\title{
Solving the inhomogeneous Bethe-Salpeter equation in Minkowski space: the zero-energy limit
}

\author{
Tobias Frederico $^{1, \mathrm{a}}$, Giovanni Salmè ${ }^{2, \mathrm{~b}}$, Michele Viviani ${ }^{3, \mathrm{c}}$ \\ ${ }^{1}$ Dep. de Física, Instituto Tecnológico de Aeronáutica, DCTA, 12.228-900 São José dos Campos, São Paulo, Brazil \\ 2 Istituto Nazionale di Fisica Nucleare, Sezione di Roma, P.le A. Moro 2, 00185 Roma, Italy \\ ${ }^{3}$ Istituto Nazionale di Fisica Nucleare, Sezione di Pisa, Largo Pontecorvo 3, 56100 Pisa, Italy
}

Received: 10 July 2015 / Accepted: 10 August 2015 / Published online: 28 August 2015

(C) The Author(s) 2015. This article is published with open access at Springerlink.com

\begin{abstract}
The inhomogeneous Bethe-Salpeter equation for an interacting system, composed of two massive scalars exchanging a massive scalar, is numerically investigated in the ladder approximation directly in Minkowski space, by using for the first time in the continuum an approach based on the Nakanishi integral representation. In this paper, the limiting case of zero-energy states is considered, thus extending an approach that has already been successfully applied to bound states. The numerical values of scattering lengths, are calculated for several values of the Yukawa coupling constant, by using two different integral equations that stem from the Nakanishi framework. Those low-energy observables are compared with (1) the analogous quantities recently obtained in literature, within a totally different framework, and (2) the non-relativistic evaluations, to illustrate the relevance of a nonperturbative, genuine field theoretical treatment in Minkowski space, even in the low-energy regime. Moreover, dynamical functions, like the Nakanishi weight functions and the distorted part of the zero-energy light-front wave functions are also presented. Interestingly, a highly non-trivial issue related to the abrupt change in the width of the support of the Nakanishi weight function, when the zero-energy limit is approached, is elucidated, ensuring a sound basis to the forthcoming evaluation of phase shifts.
\end{abstract}

\section{Introduction}

Within a field theoretical framework, it is a highly non-trivial challenge to develop nonperturbative tools in Minkowski space, but it is quite desirable to make efforts in that direction, in order to gain insights that could turn out useful in parti-

\footnotetext{
a e-mail: tobias@ita.br

b e-mail: salmeg@roma1.infn.it

c e-mail: michele.viviani@pi.infn.it
}

cle physics. In the last few years, solving the homogeneous Bethe-Salpeter equation (BSE) [1] directly in Minkowski space has allowed one to make a substantial step forward [2-11] due to approaches based on the so-called Nakanishi perturbation-theory integral representation (PTIR) of the $n$ leg transition amplitudes [12].

The Nakanishi PTIR for the three-leg amplitude is emerging as a very effective tool for studying the bound-state problem $[2-8,10,11]$, within a rigorous field-theory framework. Though the Nakanishi PTIR of the three-leg amplitude, or vertex function, had been developed within the perturbative framework of the Feynman diagrams (as is the case for any $n$-leg amplitude PTIR), it has been shown to work extremely well as the initial Ansatz for obtaining actual solutions of the homogeneous BSE. It must be recalled that the BSE, being an integral equation, belongs to a nonperturbative realm, and therefore the Nakanishi integral representation of the threeleg amplitude can be only an Ansatz when exploited in this context.

The main features of the Nakanishi integral representation of any $n$-leg amplitude are basically related to the formal infinite sum of the parametric Feynman diagrams that contribute to the amplitude under consideration. In particular, the $n$-leg amplitude PTIR has a well-defined structure, given by the folding of (1) a denominator, containing all the allowed independent invariants and governing the analytic behavior of the amplitude itself, and (2) a weight function, which is a real function depending upon real variables (one is a non-compact variable, while the others are compact). It should be emphasized that at this stage the Nakanishi weight function has only a formal expression [12]. If there were an equation for explicitly determining such a weight function, then one could quantitatively evaluate the actual $n$-leg amplitude under consideration. The homogeneous BSE, which obviously does not belong to the original framework of PTIR, has inspired a different usage of the formal expression of a particular $n$ - 
leg amplitude, namely the three-leg one, or vertex function. Indeed, if one assumes that the Nakanishi integral representation of the three-leg amplitude is formally valid also for the BS amplitude (still a three-leg amplitude, but for a bound state), then the weight function could be considered as an unknown function to be determined. It has to be pointed out that, a priori, there is no guarantee that such an approach for solving the BSE be successful, given the mentioned caveat above. Fortunately, it works, as shown in Refs. [2-8,10,11], where the above strategy was applied, with some differences, for solving the homogeneous BSE directly in Minkowski space. More precisely, by using the PTIR Ansatz for the BS amplitude one can derive, in a formally exact way, an equation for the Nakanishi weight function, starting from the homogeneous BSE, and look for solutions. If the new equation for the weight function has a solution, then one can claim that the BS amplitudes, actual solutions of the homogeneous BSE in Minkowski space, can be (1) formally written in a manner similar to the PTIR three-leg amplitude, and (2) numerically determined. In order to obtain a formally exact integral equation for the weight function from BSE, it is very useful and effective to adopt a light-front (LF) framework. This has been done both in the covariant version of the LF framework [4] and in the non-explicitly covariant one [9]. In particular, the bound states of a massive two-scalar system interacting through the exchange of a massive scalar have been studied by adopting both ladder $[4,6,7,10,11]$ and cross-ladder approximations of the BS kernel [5]. Notably, the extension to a bound fermionic system have also been undertaken [8]. It has to be recalled that numerical investigations of the homogeneous BSE has been also performed by considering the standard four-dimensional variables $[2,3]$.

The successful achievements for the homogeneous BSE encourage the extension of the Nakanishi integral representation to the study of the inhomogeneous BSE, i.e. the integral equation that determines the scattering states. Our aim is to present a new application of our general approach [9], based on the so-called LF projection of the BS amplitude, i.e. the exact integration of the minus component of the relative fourmomentum that appears in the BS amplitude. After applying this formally exact step to the BSE, we have numerically investigated the zero-energy limit of the inhomogeneous BSE, for a massive two-scalar system interacting through the exchange of a massive scalar, in the ladder approximation. The calculated scattering lengths have been compared in great detail with the analogous observables recently obtained $[13,14]$ within a completely different framework. For the sake of completeness, we have added a comparison with the non-relativistic scattering lengths. In principle, our detailed study can yield possible guidance for reducing the model dependence in the phenomenological treatment of relativistic effects in scattering states. For instance, it could contribute to an actual evaluation of the final-state interaction among light hadrons in relevant decay modes of heavy particles. The models for the final-state interactions needed in the analysis of heavy meson decay processes, like $D \rightarrow K \pi \pi[15,16]$, and in the study of $\mathrm{CP}$ violation in charmless three-body $B$ decays [17], could profit from the detailed knowledge of the two-body covariant off-shell hadronic T-matrix between mesonic states, which can be obtained by the further development of our techniques.

We have also properly analyzed the distorted part of the zero-energy wave function, putting in evidence the relation between a non-smooth behavior of the Nakanishi weight function and the expected singularities of the LF 3D wave function, like the one that contains the information relative to the global propagation of the interacting two-scalar system. Finally, the integral equation for the Nakanishi weight function obtained by applying the so-called uniqueness theorem [12], is carefully analyzed for the general case of positive energy. Such an in-depth analysis allows us to illustrate a surprising change in the width [from $(-\infty, \infty)$ to $[0, \infty)$ ] of the support of the Nakanishi weight function with respect to its non-compact variable, when the zero-energy limit is considered. Clarifying this feature allows us to put the forthcoming calculation of the phase shifts on a sound basis, since their calculation requires a careful analysis from both the theoretical and numerical points of view, as will be illustrated elsewhere [18]. To conclude this Introduction, it is useful to remind the reader that developing genuine nonperturbative descriptions of scattering processes within a Minkowski space formalism, possibly applying formally exact frameworks, is an appealing goal, in view of the aim to extract tiny, but fundamental signals once very accurate experimental data will become available.

The paper is organized as follows. In Sect. 2, we briefly introduce both the definitions and the general formalism, and we thoroughly discuss the problem of the support of the Nakanishi weight function, given its relevance for the zero-energy limit. Section 3 illustrates how to evaluate the scattering length from the Nakanishi weight function in the ladder approximation. In Sect. 4, the numerical studies of the scattering length are presented and compared with the existing calculations found in the literature; moreover, the scattering 3D LF wave function (more precisely the distorted part) is analyzed. Finally, in Sect. 5, the conclusions are drawn.

\section{The Nakanishi Integral Equations for scattering states}

In this section, (1) we quickly recall the general formalism of Ref. [9], for obtaining two integral equations that allows one to determine the Nakanishi weight function needed for scattering processes, and (2) we demonstrate a relevant feature of the weight-function support, that it turns out to be very important also for numerically solving the inhomogeneous BSE. 
In our investigation, we considered an interacting system composed by two massive scalars that exchange a massive scalar. This is a generalization of the honorable WickCutkosky model $[20,21]$ in two respects: (1) the interaction takes place through massive-scalar exchange, and (2) the scattering states is our focus.

\subsection{General formalism}

For scattering states, the incoming particles are on theirown mass-shell and we indicate their total and relative four-momenta with $p$ and $k_{i}$, respectively. By assuming that the inhomogeneous $\mathrm{BS}$ amplitude $\Phi^{+}\left(k, p, k_{i}\right)$ can be expressed in terms of the Nakanishi weight function $g^{(+)}\left(\gamma^{\prime}, z^{\prime}, z^{\prime \prime} ; \kappa^{2}, z_{i}\right)$, then one can write (cf. Ref. [9])

$$
\begin{aligned}
\Phi^{(+)}\left(k, p, k_{i}\right) & (2 \pi)^{4} \delta^{(4)}\left(k-k_{i}\right)-i \int_{-1}^{1} \mathrm{~d} z^{\prime} \int_{-1}^{1} \mathrm{~d} z^{\prime \prime} \int_{-\infty}^{\infty} \mathrm{d} \gamma^{\prime} \\
& \times \frac{g^{(+)}\left(\gamma^{\prime}, z^{\prime}, z^{\prime \prime} ; \kappa^{2}, z_{i}\right)}{\left[\gamma^{\prime}+m^{2}-\frac{1}{4} M^{2}-k^{2}-p \cdot k z^{\prime \prime}-2 k \cdot k_{i} z^{\prime}-i \epsilon\right]^{3}}= \\
= & (2 \pi)^{4} \delta^{(4)}\left(k-k_{i}\right)-i \int_{-1}^{1} \mathrm{~d} z^{\prime} \int_{-1}^{1} \mathrm{~d} z^{\prime \prime} \int_{-\infty}^{\infty} \mathrm{d} \gamma^{\prime} \\
& \times \frac{g^{(+)}\left(\gamma^{\prime}, z^{\prime}, z^{\prime \prime} ; \kappa^{2}, z_{i}\right)}{\left[\mathcal{D}_{0}-i \epsilon\right]^{3}},
\end{aligned}
$$

where the total four-momentum is $p \equiv\{M, \mathbf{0}\}$ and

$$
\begin{aligned}
\mathcal{D}_{0}= & \gamma^{\prime}+\gamma+\kappa^{2}-k^{-}\left(k^{+}+\frac{M}{2} z^{\prime \prime}-\frac{M}{2} z_{i} z^{\prime}\right) \\
& -k^{+} \frac{M}{2}\left(z^{\prime \prime}+z_{i} z^{\prime}\right)+2 z^{\prime} \cos \varphi \sqrt{\gamma \gamma_{i}} .
\end{aligned}
$$

The power of the denominator is the same one as adopted for describing a bound state (cf. Refs. [4,5,9-11]). Exploiting the standard formalism introduced in Ref. [4], one defines $z_{i}=$ $-2 k_{i}^{+} / M$ and gets $z_{i}=2 k_{i}^{-} / M$, since the incoming particles are on their-own mass shell: $\left(p / 2 \pm k_{i}\right)^{2}=m^{2}$. Moreover, one has $1 \geq\left|z_{i}\right|$, since the incoming particles have positive longitudinal momenta, i.e. $p^{+} / 2 \pm k_{i}^{+} \geq 0$. In Eq. (1), the following notations have been used: (1) $\cos \varphi=\widehat{\mathbf{k}}_{\perp} \cdot \widehat{\mathbf{k}}_{i \perp}$, (2) $\gamma=\left|\mathbf{k}_{\perp}\right|^{2}$ and $\gamma_{i}=\left|\mathbf{k}_{i \perp}\right|^{2}$, and (3) $\kappa^{2}=m^{2}-M^{2} / 4$. For the initial state one has

$$
\left(p / 2 \pm k_{i}\right)^{2}=m^{2}=\frac{M^{2}}{4}+k_{i}^{+} k_{i}^{-}-\gamma_{i}=\left(1-z_{i}^{2}\right) \frac{M^{2}}{4}-\gamma_{i},
$$

with necessarily $\left(z_{i}\right)^{2}<1$. Hence one gets

$$
\begin{aligned}
M^{2} & =4 \frac{\left(m^{2}+\gamma_{i}\right)}{\left(1-z_{i}^{2}\right)} \\
\kappa^{2} & =-\gamma_{i}-z_{i}^{2} \frac{M^{2}}{4}=k_{i}^{2} \leq 0 .
\end{aligned}
$$

To complete the generalities, we also give the expression for the inhomogeneous BSE, without self-energy insertions and vertex corrections, in the present stage of our approach. Then one can write

$$
\begin{aligned}
& \Phi^{(+)}\left(k, p, k_{i}\right)=(2 \pi)^{4} \delta^{(4)}\left(k-k_{i}\right) \\
& \quad+G_{0}^{(12)}(k, p) \int \frac{\mathrm{d}^{4} k^{\prime}}{(2 \pi)^{4}} i \mathcal{K}\left(k, k^{\prime}, p\right) \Phi^{(+)}\left(k^{\prime}, p, k_{i}\right),
\end{aligned}
$$

where $i \mathcal{K}$ is the interaction kernel (where the vertex corrections should appear), and $G_{0}^{(12)}$ is the free two-particle Green's function given by

$$
\begin{aligned}
& G_{0}^{(12)}(k, p)=G_{0}^{(1)} G_{0}^{(2)} \\
& =\frac{i}{\left(\frac{p}{2}+k\right)^{2}-m^{2}+i \epsilon} \frac{i}{\left(\frac{p}{2}-k\right)^{2}-m^{2}+i \epsilon} .
\end{aligned}
$$

It is worth noting that the bosonic symmetry of the BS amplitude, Eq. (1), when $1 \rightarrow 2$ (i.e. $p \rightarrow p, k \rightarrow(-k)$ and $\left.k_{i} \rightarrow\left(-k_{i}\right)\right)$ has to be fulfilled, as in the case of bound states [10]. Therefore, the Nakanishi weight function must have the following property:

$g^{(+)}\left(\gamma^{\prime}, z^{\prime}, z^{\prime \prime} ; \kappa^{2}, z_{i}\right)=g^{(+)}\left(\gamma^{\prime}, z^{\prime},-z^{\prime \prime} ; \kappa^{2},-z_{i}\right)$.

Moreover, as shown in detail in Appendix A one has

$g^{(+)}\left(\gamma^{\prime}, z^{\prime}= \pm 1, z^{\prime \prime}\right)=g^{(+)}\left(\gamma^{\prime}, z^{\prime}, z^{\prime \prime}= \pm 1\right)=0$.

As is well known (see e.g. Refs. [9,22,23]), by projecting the BS amplitude onto the null-plane, i.e. integrating on $k^{-}$, one exactly gets the 3D LF scattering wave function $\psi^{(+)}$, which is proportional to the valence component $\psi_{n=2 / p}^{(+)}$appearing in the Fock expansion of a two-scalar state, namely $\psi^{(+)}=$ $\sqrt{2} \psi_{n=2 / p}^{(+)}$(given the normalizations assumed in Refs. [9, 10]). The 3D LF scattering wave function reads

$$
\begin{aligned}
\psi^{(+)} & \left(z, \gamma, \cos \varphi ; \kappa^{2}, z_{i}\right) \\
= & p^{+} \frac{\left(1-z^{2}\right)}{4} \int \frac{\mathrm{d} k^{-}}{2 \pi} \Phi^{(+)}\left(k, p, k_{i}\right)=p^{+} \frac{\left(1-z^{2}\right)}{4} \\
& \times(2 \pi)^{3} \delta^{(3)}\left(\tilde{k}-\tilde{k}_{i}\right)+\psi_{\text {dist }}\left(z, \gamma, \cos \varphi ; \kappa^{2}, z_{i}\right)
\end{aligned}
$$

where $\tilde{k} \equiv\left\{k^{+}, \mathbf{k}_{\perp}\right\}$ and $\psi_{\text {dist }}\left(z, \gamma, \cos \varphi ; \kappa^{2}, z_{i}\right)$ is the distorted part of the 3D LF scattering wave function, which in the CM frame, where $p^{+}=p^{-}=M / 2$ and $\mathbf{p}_{\perp}=0$, reads

$$
\begin{aligned}
& \psi_{\text {dist }}\left(z, \gamma, \cos \varphi ; \kappa^{2}, z_{i}\right)=\frac{\left(1-z^{2}\right)}{4} \int_{-1}^{1} \mathrm{~d} z^{\prime} \\
& \times \int_{-\infty}^{\infty} \mathrm{d} \gamma^{\prime} \frac{g^{(+)}\left(\gamma^{\prime}, z^{\prime}, z ; \kappa^{2}, z_{i}\right)}{\left[\mathcal{D}_{1}-i \epsilon\right]^{2}}
\end{aligned}
$$

with

$$
\begin{aligned}
\mathcal{D}_{1}= & \gamma^{\prime}+\gamma+z^{2} m^{2}+\left(1-z^{2}\right) \kappa^{2} \\
& +z^{\prime}\left(\frac{M^{2}}{2} z z_{i}+2 \cos \varphi \sqrt{\gamma \gamma_{i}}\right) .
\end{aligned}
$$


In what follows, without loss of generality, we choose a head-on scattering process, namely a $z$-axis along the incoming three-momenta. In this case the variable $\gamma_{i}$ is zero and therefore the dependence upon $\cos \varphi$ disappears. As a matter of fact, the distorted wave function becomes

$$
\begin{aligned}
\psi_{\text {dist }}\left(z, \gamma ; \kappa^{2}, z_{i}\right)= & \frac{\left(1-z^{2}\right)}{4} \int_{-1}^{1} \mathrm{~d} z^{\prime} \int_{-\infty}^{\infty} \mathrm{d} \gamma^{\prime} \\
& \times \frac{g^{(+)}\left(\gamma^{\prime}, z^{\prime}, z ; \kappa^{2}, z_{i}\right)}{\left[\mathcal{D}_{2}-i \epsilon\right]^{2}}
\end{aligned}
$$

with

$\mathcal{D}_{2}=\gamma^{\prime}+\gamma+z^{2} m^{2}+\left(1-z^{2}\right) \kappa^{2}+z^{\prime} \frac{M^{2}}{2} z z_{i}$

and

$z_{i}= \pm \frac{2}{M} \sqrt{-\kappa^{2}}$

Remarkably, $\psi_{\text {dist }}\left(z, \gamma ; \kappa^{2}, z_{i}\right)$ displays a cut, originating by the free propagation of the two constituents, just as in the non-relativistic case. In particular, the distorted part of the scattering wave function can be rearranged in order to make explicit the free propagation, obtaining (see details in Appendix B)

$$
\begin{aligned}
& \psi_{\text {dist }}\left(z, \gamma ; \kappa^{2}, z_{i}\right)=i \frac{\left(1-z^{2}\right)}{4} \\
& \times \frac{1}{\left[\kappa^{2}\left(1-z^{2}\right)+m^{2} z^{2}+\gamma-i \epsilon\right]} \int_{-1}^{1} \mathrm{~d} \zeta^{\prime \prime} \int_{-1}^{1} \mathrm{~d} \zeta^{\prime} \\
& \times \int_{-\infty}^{\infty} \mathrm{d} \gamma^{\prime \prime} \widetilde{\mathcal{G}}^{+}\left(\gamma^{\prime \prime}, \zeta^{\prime \prime}, \zeta^{\prime} ; \kappa^{2}, z_{i}\right) \theta\left(1-\left|\zeta^{\prime \prime}\right|-\left|\zeta^{\prime}\right|\right) \\
& \times\left[\frac{(1+z)}{\left(1+\zeta^{\prime}-\zeta^{\prime \prime} z_{i}\right)} \frac{\theta\left(\zeta^{\prime}-z-\zeta^{\prime \prime} z_{i}\right)}{\mathcal{D}_{3}\left(z, \zeta^{\prime}, \zeta^{\prime \prime}\right)-i \epsilon}\right. \\
& \left.+\frac{(1-z)}{\left(1-\zeta^{\prime}+\zeta^{\prime \prime} z_{i}\right)} \frac{\theta\left(z+\zeta^{\prime \prime} z_{i}-\zeta^{\prime}\right)}{\mathcal{D}_{3}\left(-z,-\zeta^{\prime},-\zeta^{\prime \prime}\right)-i \epsilon}\right]
\end{aligned}
$$

where

$$
\begin{aligned}
\mathcal{D}_{3}\left(z, \zeta^{\prime}, \zeta^{\prime \prime}\right)= & \kappa^{2}\left(1-z^{2}\right)+m^{2} z^{2}+\gamma \\
& +\frac{(1+z)}{\left(1+\zeta^{\prime}-\zeta^{\prime \prime} z_{i}\right)}\left(\frac{M^{2}}{2} z \zeta^{\prime \prime} z_{i}+\gamma^{\prime \prime}\right)
\end{aligned}
$$

and $\widetilde{\mathcal{G}}^{+}\left(\gamma^{\prime}, \zeta^{\prime \prime}, \zeta^{\prime} ; \kappa^{2}, z_{i}\right)$ is the Nakanishi weight function for the half-off-shell $T$-matrix (see Ref. [9]). In particular, the relation between the two Nakanishi weight functions is given by

$$
\begin{aligned}
g^{+} & \left(\gamma^{\prime}, z^{\prime}, z ; \kappa^{2}, z_{i}\right) \\
= & i \int_{0}^{1} \frac{\mathrm{d} \alpha}{\alpha^{3}} \int_{-1}^{1} \mathrm{~d} \zeta^{\prime} \widetilde{\mathcal{G}}^{+}\left(\frac{\gamma^{\prime}}{\alpha}, \frac{z^{\prime}}{\alpha}, \frac{\zeta^{\prime}}{a} ; \kappa^{2}, z_{i}\right) \\
& \times \theta\left(\alpha-\left|z^{\prime}\right|-\left|\zeta^{\prime}\right|\right) \theta\left(1-\alpha-\left|\zeta^{\prime}-z-z^{\prime} z_{i}\right|\right),
\end{aligned}
$$

with all the constraints on the variables explicitly written. Indeed, notice that the dependence upon $z$ in the weight function $g_{(L d)}^{(+)}\left(\gamma^{\prime}, z^{\prime}, z ; \kappa^{2}, z_{i}\right)$ should be read as $z+z^{\prime} z_{i}$ (cf. Eq. (66) in [9] and Appendix B of the present paper). From Eq. (12), the analogy with the non relativistic case appears evident, once the familiar form of the global propagation is recognized. As a matter of fact, one has $\left(1>z^{2}\right)$

$\frac{1}{\gamma+z^{2} m^{2}+\left(1-z^{2}\right) \kappa^{2}-i \epsilon}=\frac{\left(1-z^{2}\right)}{4} \frac{1}{M_{0}^{2}-M^{2}-i \epsilon}$,

where $M_{0}$ is the free mass of the two-body system given by

$M_{0}^{2}=4 \frac{\left(m^{2}+\gamma\right)}{\left(1-z^{2}\right)}$.

It should be pointed out that the cut in $\psi_{\text {dist }}$ is mirrored in the integral equation determining the Nakanishi weight function, in particular in the part governed by the dynamics [see Eq. (18), below]. It is useful to anticipate that the cut is canceled by the proper factor in the evaluation of the scattering amplitude.

An issue of fundamental relevance related to $\psi_{\text {dist }}$ in Eq. (10) [or to $\psi_{\text {dist }}$ in Eq. (12)] is to determine the support of the Nakanishi weight function $g^{(+)}\left(\gamma^{\prime}, z^{\prime}, z ; \kappa^{2}, z_{i}\right)$ (or equivalently $\left.\widetilde{\mathcal{G}}^{+}\left(\gamma^{\prime}, z^{\prime}, \zeta^{\prime} ; \kappa^{2}, z_{i}\right)\right)$ with respect to the non-compact variable $\gamma^{\prime}$. While the variable $\gamma=k_{\perp}^{2}$ in $\psi_{\text {dist }}\left(z, \gamma ; \kappa^{2}, z_{i}\right)$ is such that $\gamma \in[0, \infty)$ and the same holds for $\gamma^{\prime}$ in the Nakanishi weight function when the bound state is discussed (see [10]), in the case of a scattering state one has a different interval, namely $\gamma^{\prime} \in(-\infty, \infty)$. Then a question arises about the width of the support when $\kappa^{2} \rightarrow 0^{-}$, i.e. the zeroenergy limit which we are interested in. One should expect that the relevant support of $\gamma^{\prime}$ had to shrink in order to match the one pertaining to a bound state.

This can be accomplished if

$\lim _{\kappa^{2} \rightarrow 0^{-}} g^{(+)}\left(\gamma^{\prime}, z^{\prime}, z ; \kappa^{2}, z_{i}\right)=0$

for $\gamma^{\prime}<0$. Notably, this is what happens, as shown in detail in the following subsection. It should be pointed out that such a result is relevant for what follows, since we are going to consider the limit of a scattering state for $\kappa^{2} \rightarrow 0^{-}$, and one could be puzzled by the abrupt transition of the lower extremum for $\gamma^{\prime}$ from an unbound value, for $\kappa^{2}<-\epsilon$, to a bound one, for the zero-energy limit.

2.2 The support of the Nakanishi weight function for the inhomogeneous BSE

In order to address the support issue above introduced, let us consider the first meaningful approximation to Eq. (4), namely the approximation where the kernel $i \mathcal{K}$ is substituted by its ladder contribution, given by 
$i \mathcal{K}^{(L d)}\left(k, k_{i}, p\right)=i \frac{(-i g)^{2}}{\left(k-k^{\prime}\right)^{2}-\mu^{2}+i \epsilon}$.

First, one inserts the Nakanishi Ansatz for the BS amplitude, Eq. (1), in the ladder BSE. Then one can perform the integration over $k^{-}$without any approximation, and obtain the ladder inhomogeneous BSE projected onto the null-plane, i.e. an integral equation that relates $\psi_{\text {dist }}$ given by Eq. (10), to the dynamics dictated by the ladder kernel (see details in Ref. [9]). Namely, one gets

$$
\begin{aligned}
& \int_{-\infty}^{\infty} \mathrm{d} \gamma^{\prime} \int_{-1}^{1} \mathrm{~d} z^{\prime} \frac{g_{(L d)}^{(+)}\left(\gamma^{\prime}, z^{\prime}, z ; \kappa^{2}, z_{i}\right)}{\left[\mathcal{D}_{2}-i \epsilon\right]^{2}} \\
& =\frac{g^{2}}{\left[\gamma+z^{2} m^{2}+\left(1-z^{2}\right) \kappa^{2}-i \epsilon\right]}\left[\mathcal{W}^{(L d)}\left(\gamma, z ; \kappa^{2}, z_{i}\right)\right. \\
& \quad+\frac{1}{2(4 \pi)^{2}} \int_{-\infty}^{\infty} \mathrm{d} \gamma^{\prime} \int_{-1}^{1} \mathrm{~d} \zeta \int_{-1}^{1} \mathrm{~d} \zeta^{\prime} g_{(L d)}^{(+)}\left(\gamma^{\prime}, \zeta, \zeta^{\prime} ; \kappa^{2}, z_{i}\right) \\
& \left.\quad \times \int_{0}^{\infty} \mathrm{d} y F\left(y, \gamma, z ; \gamma^{\prime}, \zeta, \zeta^{\prime}\right)\right],
\end{aligned}
$$

where $\mathcal{W}^{(L d)}$ is

$$
\begin{aligned}
& \mathcal{W}^{(L d)}\left(\gamma, z ; \kappa^{2}, z_{i}\right)=\frac{1}{\left(z-z_{i}\right)} \\
& \times\left\{\frac{\theta\left(z-z_{i}\right)}{\frac{M_{0}^{2}}{4}(1+z)-\frac{M^{2}}{4}\left(1+z_{i}\right)+\frac{\mu^{2}+\gamma}{\left(z-z_{i}\right)}-i \epsilon}\right. \\
& \left.-\frac{\theta\left(z_{i}-z\right)}{\frac{M_{0}^{2}}{4}(1-z)-\frac{M^{2}}{4}\left(1-z_{i}\right)+\frac{\mu^{2}+\gamma}{\left(z_{i}-z\right)}-i \epsilon}\right\},
\end{aligned}
$$

and $F$ is

$$
\begin{aligned}
& F\left(y, \gamma, z ; \gamma^{\prime}, \zeta, \zeta^{\prime}\right)=\frac{(1+z)^{2}}{\left(1+\zeta^{\prime}-z_{i} \zeta\right)^{2}} \\
& \quad \times \frac{\theta\left(\zeta^{\prime}-z-z_{i} \zeta\right)}{\left[\mathcal{D}_{4}\left(y, \gamma, z ; \gamma^{\prime}, \zeta, \zeta^{\prime} ; z_{i}\right)-i \epsilon\right]^{2}}+\frac{(1-z)^{2}}{\left(1-\zeta^{\prime}+z_{i} \zeta\right)^{2}} \\
& \quad \times \frac{\theta\left(z+z_{i} \zeta-\zeta^{\prime}\right)}{\left[\mathcal{D}_{4}\left(y, \gamma,-z ; \gamma^{\prime}, \zeta,-\zeta^{\prime} ;-z_{i}\right)-i \epsilon\right]^{2}}
\end{aligned}
$$

where

$$
\begin{gathered}
\mathcal{D}_{4}\left(y, \gamma, z ; \gamma^{\prime}, \zeta, \zeta^{\prime} ; z_{i}\right)=\gamma+z^{2} m^{2}+\kappa^{2}\left(1-z^{2}\right) \\
+\Gamma\left(y, z, z_{i}, \zeta, \zeta^{\prime}, \gamma^{\prime}\right)+Z\left(z, \zeta, \zeta^{\prime} ; z_{i}\right) \frac{M^{2}}{2} z z_{i}
\end{gathered}
$$

with

$$
\begin{aligned}
& \Gamma\left(y, z, z_{i}, \zeta, \zeta^{\prime}, \gamma^{\prime}\right)=\frac{(1+z)}{\left(1+\zeta^{\prime}-z_{i} \zeta\right)} \\
& \times\left\{y \mathcal{A}\left(\zeta, \zeta^{\prime}, \gamma^{\prime}, \kappa^{2}\right)+\frac{\mu^{2}}{y}+\mu^{2}+\gamma^{\prime}\right\} \\
& Z\left(z, \zeta, \zeta^{\prime} ; z_{i}\right)=\frac{(1+z)}{\left(1+\zeta^{\prime}-z_{i} \zeta\right)} \zeta,
\end{aligned}
$$

and

$$
\mathcal{A}\left(\zeta, \zeta^{\prime}, \gamma^{\prime}, \kappa^{2}\right)=\zeta^{\prime 2} \frac{M^{2}}{4}+\kappa^{2}\left(1+\zeta^{2}\right)+\gamma^{\prime}
$$

Because of the presence of the theta functions in Eq. (20), one has

$$
1 \geq|\zeta| \geq\left|Z\left( \pm z, \zeta, \pm \zeta^{\prime}, z_{i}\right)\right|
$$

It should be pointed out that Eq. (18) is relevant for the calculation of the phase shifts and, in the zero-energy limit, of the scattering lengths (cf. Sect. 3).

After combining the global propagation with the denominator in $\mathcal{W}^{(L d)}\left(\gamma, z ; \kappa^{2}, z_{i}\right)$ and repeating the same step for $F$ (see Ref. [9]) one can apply the Nakanishi theorem on the uniqueness of the weight function for an $n$-leg transition amplitude [12]. It should be recalled that the uniqueness theorem has been proven within a perturbative framework, while in the present context, a nonperturbative one, the uniqueness is conjectured and numerically checked. Eventually, one gets a new integral equation for the Nakanishi weight function, which allows us to discuss the support issue and to understand the sharp transition from the bound case to the scattering one (see Appendix C). The integral equation from the uniqueness theorem reads (see [9])

$$
\begin{aligned}
& g_{(L d)}^{(+)}\left(\gamma, z^{\prime}, z ; \kappa^{2}, z_{i}\right)=g^{2} \theta\left(-z^{\prime}\right) \delta\left(\gamma-\gamma_{a}\left(z^{\prime}\right)\right) \\
& \quad \times\left\{\theta\left(z-z_{i}\right) \theta\left[1-z+z^{\prime}\left(1-z_{i}\right)\right]\right. \\
& \left.\quad+\theta\left(z_{i}-z\right) \theta\left[1+z+z^{\prime}\left(1+z_{i}\right)\right]\right\} \\
& \quad-\frac{g^{2}}{2(4 \pi)^{2}} \int_{-\infty}^{\infty} \mathrm{d} \gamma^{\prime} \int_{-1}^{1} \mathrm{~d} \zeta \int_{-1}^{1} \mathrm{~d} \zeta^{\prime} g_{(L d)}^{(+)}\left(\gamma^{\prime}, \zeta, \zeta^{\prime} ; \kappa^{2}, z_{i}\right) \\
& \quad \times\left[\frac{(1+z) \theta\left(\zeta^{\prime}-z-z_{i} \zeta\right)}{\left(1+\zeta^{\prime}-z_{i} \zeta\right)} h^{\prime}\left(\gamma, z^{\prime}, z, z_{i} ; \gamma^{\prime}, \zeta, \zeta^{\prime}, \mu^{2}\right)\right. \\
& \left.\quad+\frac{(1-z) \theta\left(z-\zeta^{\prime}+z_{i} \zeta\right)}{\left(1-\zeta^{\prime}+z_{i} \zeta\right)} h^{\prime}\left(\gamma, z^{\prime},-z,-z_{i} ; \gamma^{\prime}, \zeta,-\zeta^{\prime}, \mu^{2}\right)\right]
\end{aligned}
$$

where

$\gamma_{a}\left(z^{\prime}\right)=z^{\prime}\left(2 \kappa^{2}-\mu^{2}\right) \geq 0$

and $h^{\prime}\left(\gamma^{\prime \prime}, z^{\prime}, z, z_{i} ; \gamma^{\prime}, \zeta, \zeta^{\prime}, \mu^{2}\right)$ is given by

$$
\begin{aligned}
& h^{\prime}\left(\gamma^{\prime \prime}, z^{\prime}, z, z_{i} ; \gamma^{\prime}, \zeta, \zeta^{\prime}, \mu^{2}\right)=\frac{(1+z)}{\left(1+\zeta^{\prime}-z_{i} \zeta\right)} \\
& \quad \times\left\{\frac{\partial}{\partial \lambda} \int_{0}^{\infty} \mathrm{d} y \int_{0}^{1} \mathrm{~d} \xi \delta\left[z^{\prime}-\xi Z\left(z, \zeta, \zeta^{\prime} ; z_{i}\right)\right]\right. \\
& \left.\quad \times \delta\left[\mathcal{F}\left(\lambda, y, \xi ; \gamma^{\prime \prime}, z, \zeta, \zeta^{\prime}, \gamma^{\prime} ; z_{i}, \kappa^{2}, \mu^{2}\right)\right]\right\}_{\lambda=0}
\end{aligned}
$$


with

$$
\begin{aligned}
\mathcal{F} & \left(\lambda, y, \xi ; \gamma^{\prime \prime}, z, \zeta, \zeta^{\prime}, \gamma^{\prime} ; z_{i}, \kappa^{2}, \mu^{2}\right) \\
= & \gamma^{\prime \prime}-\xi \frac{(1+z)}{\left(1+\zeta^{\prime}-z_{i} \zeta\right)} \\
& \times\left(\frac{y^{2} \mathcal{A}\left(\zeta, \zeta^{\prime}, \gamma^{\prime}, \kappa^{2}\right)+y\left(\mu^{2}+\gamma^{\prime}\right)+\mu^{2}}{y}\right)-\xi \lambda .
\end{aligned}
$$

Notice that the inhomogeneous term vanishes both at $z= \pm 1$ and at $z^{\prime}=-1$, as expected [cf. Eq. (7)]. Indeed, for $z=1$, one has

$$
\begin{array}{r}
\theta\left(-z^{\prime}\right)\left\{\theta\left(1-z_{i}\right) \theta\left[z^{\prime}\left(1-z_{i}\right)\right]\right. \\
\left.+\theta\left(z_{i}-1\right) \theta\left[2+z^{\prime}\left(1+z_{i}\right)\right]\right\},
\end{array}
$$

which vanishes. For $z=-1$, one gets

$$
\begin{aligned}
& \theta\left(-z^{\prime}\right)\left\{\theta\left(-1-z_{i}\right) \theta\left[z^{\prime}\left(1-z_{i}\right)\right]\right. \\
& \left.\quad+\theta\left(z_{i}+1\right) \theta\left[z^{\prime}\left(1+z_{i}\right)\right]\right\},
\end{aligned}
$$

and again the theta functions produce a vanishing outcome. Finally, if $z^{\prime}=-1$, the inhomogeneous term is vanishing, since

$$
\begin{aligned}
& \theta\left(z-z_{i}\right) \theta\left[1-z-\left(1-z_{i}\right)\right] \\
& \quad+\theta\left(z_{i}-z\right) \theta\left[1+z-\left(1+z_{i}\right)\right]=0 .
\end{aligned}
$$

For $\kappa^{2}<0$ the support of $g_{(L d)}^{(+)}$with respect to the variable $\gamma$ is the open interval $(-\infty, \infty)$, and one can usefully split the integral equation (26) in two coupled integral equations: one is inhomogeneous, while the other is homogeneous. To achieve this goal, one can adopt the following decomposition:

$$
\begin{aligned}
& g_{(L d)}^{(+)}\left(\gamma, z^{\prime}, z ; \kappa^{2}, z_{i}\right)=\theta(\gamma) g_{p ;(L d)}^{(+)}\left(\gamma, z^{\prime}, z ; \kappa^{2}, z_{i}\right) \\
& \quad+\theta(-\gamma) g_{n ;(L d)}^{(+)}\left(\gamma, z^{\prime}, z ; \kappa^{2}, z_{i}\right) .
\end{aligned}
$$

If $\kappa^{2} \rightarrow 0^{-}$, the off-shell kernel in the homogeneous integral equation (see details in Appendix C), becomes vanishing and this leads to a system of uncoupled equations. In this case, the homogeneous integral equation, is expected to have as a solution only $g_{n ;(L d)}^{(+)}\left(\gamma, z^{\prime}, z ; \kappa^{2}=z_{i}=0\right)=0$, given the freedom in choosing $g^{2}$ for scattering states. Let us recall that for the bound-state case, where $\kappa^{2} \geq 0$ and $\gamma>0$, one gets a homogeneous integral equation and deals with a proper eigenvalue problem. In particular, one finds a discrete spectrum for $g^{2}$, once a value is assigned to $\kappa^{2}$ and $\mu$ (see, e.g., Ref. [10], where the bound-state case is discussed, within the present approach).

In conclusion, for scattering states in the limit $\kappa^{2} \rightarrow 0^{-}$, the corresponding Nakanishi weight function reduces to only the component $g_{p ;(L d)}^{(+)}\left(\gamma, z^{\prime}, z ; \kappa^{2}=z_{i}=0\right)$ and fulfills the following inhomogeneous integral equation:

$$
\begin{aligned}
& g_{p ;(L d)}^{(+)}\left(\gamma, z^{\prime}, z ; \kappa^{2}=z_{i}=0\right)=g^{2} \theta\left(-z^{\prime}\right) \delta\left(\gamma-\gamma_{a}\left(z^{\prime}\right)\right) \\
& \quad \times\left\{\theta(z) \theta\left[1-z+z^{\prime}\right]+\theta(-z) \theta\left[1+z+z^{\prime}\right]\right\} \\
& \quad-\frac{g^{2}}{2(4 \pi)^{2}} \theta(\gamma) \int_{0}^{\infty} \mathrm{d} \gamma^{\prime} \int_{-1}^{1} \mathrm{~d} \zeta \\
& \quad \times \int_{-1}^{1} \mathrm{~d} \zeta^{\prime} H^{\prime}\left(\gamma, z^{\prime}, z, z_{i}=0 ; \gamma^{\prime}, \zeta, \zeta^{\prime}, \mu^{2}\right) \\
& \quad \times g_{p ;(L d)}^{(+)}\left(\gamma^{\prime}, \zeta, \zeta^{\prime} ; \kappa^{2}=z_{i}=0\right)
\end{aligned}
$$

This is sharply different from the general case $\kappa^{2}<0$, given by Eqs. (C.25) and (C.26) in Appendix C.

\section{The scattering length}

In the CM frame, the differential cross section for the elastic scattering of two scalars can be written as follows [19]:

$\frac{\mathrm{d} \sigma}{\mathrm{d} \Omega}=\frac{1}{64 \pi^{2} s}\left|T_{i i}^{e l}\right|^{2}=\left|f^{e l}(s, \theta)\right|^{2}$,

with $T_{i i}^{e l}$ the invariant matrix element of the T-matrix, that is dimensionless (recall that in a $\phi^{3}$ theory the coupling constant $g$ has the dimension of a mass), and $f^{e l}(s, \theta)$ the elastic scattering amplitude. It turns out that

$f^{e l}(s, \theta)=-\frac{1}{8 \pi \sqrt{s}} T_{i i}^{e l}$,

where $s=M^{2}$ and $\cos \theta=\hat{k}_{f} \cdot \hat{k}_{i}$. To introduce the relation with the phase shifts $\delta_{\ell}$, let us expand the scattering amplitude on the basis of the Legendre polynomials, $P_{\ell}(\cos \theta)$, as follows:

$f^{e l}(s, \theta)=\frac{1}{k_{r}} \sum_{\ell}(2 \ell+1) f_{\ell}^{e l} P_{\ell}(\cos \theta)$,

where the relative three-momentum is $k_{r}=\sqrt{s / 4-m^{2}}$, or $k_{r}^{2}=-\kappa^{2}$, and the projected amplitudes are given by

$f_{\ell}^{e l}=e^{i \delta_{\ell}} \sin \delta_{\ell}$.

Finally, in the zero-energy limit, only the amplitude with $\ell=0$ survives and one obtains

$f_{0}^{e l} \simeq \delta_{0} \simeq-a k_{r}$,

where $a$ is the s-wave scattering length. Therefore, in the zero-energy limit one gets

$\lim _{s \rightarrow 4 m^{2}} f^{e l}(s, \theta)=-a$

On the other hand, the scattering amplitude can be calculated through the BS amplitude as follows (see Ref. [9] for details): 


$$
\begin{aligned}
& f^{e l}(s, \theta)=-\frac{i}{\sqrt{s} 8 \pi} \lim _{k^{\prime} \rightarrow k_{f}}\left\langle k^{\prime}, p\left|G_{0}^{-1}(p)\right| \Phi^{(+)} ; p, k_{i}\right\rangle= \\
& =\frac{1}{\sqrt{s} 8 \pi} \lim _{(\gamma, z) \rightarrow\left(\gamma_{f}, z_{f}\right)}\left[\gamma+z^{2} m^{2}+\left(1-z^{2}\right) \kappa^{2}-i \epsilon\right] \\
& \quad \times \frac{4}{\left(1-z^{2}\right)} \psi_{\text {dist }}\left(z, \gamma ; \kappa^{2}, z_{i}\right),
\end{aligned}
$$

where $k^{\prime}=\left(p_{1}^{\prime}-p_{2}^{\prime}\right) / 2, p_{1}^{\prime}+p_{2}^{\prime}=p$ (recall that $p^{2}=$ $M^{2}=s$ ).

In the ladder approximation and choosing $\gamma_{i}=0$, from Eqs. (10) and (18) one gets

$$
\begin{aligned}
& f_{(L d)}^{e l}(s, \theta)=\frac{2 \alpha m^{2}}{\sqrt{s}}\left[\mathcal{W}^{(L d)}\left(\gamma_{f}, z_{f} ; \kappa^{2}, z_{i}\right)\right. \\
& +\frac{1}{2(4 \pi)^{2}} \int_{-\infty}^{\infty} \mathrm{d} \gamma^{\prime \prime} \int_{-1}^{1} \mathrm{~d} \zeta \int_{-1}^{1} \mathrm{~d} \zeta^{\prime} \\
& \left.\quad \times \int_{0}^{\infty} \mathrm{d} y F\left(y, \gamma_{f}, z_{f} ; \gamma^{\prime \prime}, \zeta, \zeta^{\prime}\right) g_{(L d)}^{(+)}\left(\gamma^{\prime \prime}, \zeta, \zeta^{\prime} ; \kappa^{2}, z_{i}\right)\right]
\end{aligned}
$$

where

$\alpha=\frac{g^{2}}{m^{2} 16 \pi}$.

If $z \rightarrow \pm 1$ the free mass $M_{0}^{2} \rightarrow \infty$, and one can see that $\mathcal{W}^{(L d)}\left(\gamma, z= \pm 1 ; \kappa^{2}, z_{i}\right) \rightarrow 0$, by taking into account also the constraints generated by the theta functions. In general, the denominators in Eq. (19) do not vanish, since (1) only minus components of on-mass-shell particles are present there, and (2) the momentum conservation law does not hold for those components (one can also explicitly check that the denominators do not have real roots). Moreover, since $\gamma_{i}=0$ then $M^{2}=m^{2} / z_{i}=\left(m^{2}+\gamma_{f}\right) / z_{f}$. It is useful to introduce some kinematical relations relevant for describing the scattering process. In particular, the initial and final Cartesian three-momenta, $\mathbf{k}_{i}$ and $\mathbf{k}_{f}$, has to be completed giving the third components, viz.

$$
\begin{aligned}
& k_{i z}=\frac{1}{2}\left(k_{i}^{+}-k_{i}^{-}\right)=-z_{i} \frac{M}{2}, \\
& k_{f z}=\frac{1}{2}\left(k_{f}^{+}-k_{f}^{-}\right)=-z_{f} \frac{M}{2} .
\end{aligned}
$$

Then one can write down the relation between the scattering angle $\theta$ and the LF variables $z_{f}$ and $z_{i}$, given by

$\mathbf{k}_{i} \cdot \mathbf{k}_{f}=z_{i} z_{f} \frac{M^{2}}{4}=-\kappa^{2} \cos \theta$,

where $p \cdot k_{i}=p \cdot k_{f}=0$ has been used (those constraints are imposed by the on-mass-shellness of the particles in the elastic channel). It also follows that

$$
\left|\mathbf{k}_{i}\right|^{2}=\left|\mathbf{k}_{f}\right|^{2}=-\kappa^{2} .
$$

Finally, by exploiting the relation $\kappa^{2}=-z_{i}^{2} M^{2} / 4$, which holds for $\gamma_{i}=0$, one gets

$z_{f}=z_{i} \cos \theta$.

For $\kappa^{2}=\left(m^{2}-s / 4\right) \rightarrow 0^{-}$, both $z_{i}$ and $z_{f}$ vanish (as well as $\gamma_{f}=\left|k_{f \perp}\right|^{2}$ ), and one loses the dependence upon the scattering angle $\theta$ in the scattering amplitude, namely one has a s-wave scattering, as it must be. The two functions, $\mathcal{W}^{(L d)}\left(\gamma_{f}, z_{f} ; \kappa^{2}, z_{i}\right)$ and $F\left(y, \gamma_{f}, z_{f} ; \gamma^{\prime \prime}, \zeta, \zeta^{\prime}\right)$, become

$$
\begin{aligned}
\lim _{\kappa^{2} \rightarrow 0^{-}} & \mathcal{W}^{(L d)}\left(\gamma_{f}, z_{f}=z_{i} \cos \theta ; \kappa^{2}, z_{i}\right) \\
= & \lim _{\kappa^{2} \rightarrow 0^{-}} \frac{1}{\left(z_{f}-z_{i}\right)}\left\{\frac{\theta\left(z_{f}-z_{i}\right)}{\frac{M^{2}}{4}\left(z_{f}-z_{i}\right)+\frac{\mu^{2}+\gamma_{f}}{\left(z_{f}-z_{i}\right)}-i \epsilon}\right. \\
& \left.+\frac{\theta\left(z_{i}-z_{f}\right)}{-\frac{M^{2}}{4}\left(z_{i}-z_{f}\right)-\frac{\mu^{2}+\gamma_{f}}{\left(z_{i}-z_{f}\right)}+i \epsilon}\right\}=\frac{1}{\mu^{2}}
\end{aligned}
$$

and

$$
\begin{aligned}
& \lim _{\kappa^{2} \rightarrow 0^{-}} F\left(y, \gamma_{f}, z_{f}=z_{i} \cos \theta ; \gamma^{\prime \prime}, \zeta, \zeta^{\prime}\right) \\
& =\frac{y^{2}}{\left[y^{2}\left(m^{2} \zeta^{\prime 2}+\gamma^{\prime \prime}\right)+y\left(\mu^{2}+\gamma^{\prime \prime}\right)+\mu^{2}-i \epsilon\right]^{2}} .
\end{aligned}
$$

Then, in the zero-energy limit, Eq. (42) reduces to (see also Appendix D)

$$
\begin{aligned}
\lim _{s \rightarrow 4 m^{2}} f_{0 L d}^{e l}(s, \theta)=-a \\
=m \alpha\left\{\frac{1}{\mu^{2}}+\frac{1}{2(4 \pi)^{2}} \int_{0}^{\infty} \mathrm{d} \gamma^{\prime \prime} \int_{-1}^{1} \mathrm{~d} \zeta^{\prime} g_{0 L d}^{(+)}\left(\gamma^{\prime \prime}, \zeta^{\prime}\right)\right. \\
\left.\quad \times \int_{0}^{\infty} d y \frac{y^{2}}{\left[y^{2} \mathcal{A}_{0}\left(\zeta^{\prime}, \gamma^{\prime \prime}\right)+y\left(\mu^{2}+\gamma^{\prime \prime}\right)+\mu^{2}-i \epsilon\right]^{2}}\right\},
\end{aligned}
$$

where the first term in the curly brackets leads to the scattering length in Born approximation, viz.

$a_{B A}=-m \frac{\alpha}{\mu^{2}}$.

Moreover, $g_{0 L d}^{(+)}\left(\gamma^{\prime \prime}, \zeta^{\prime}\right)$ is the Nakanishi weight function in the zero-energy limit. It can be obtained by solving two different integral equations as discussed in detail in Appendix $\mathrm{D}$, where the whole matter is presented in a substantially simpler way than the one in Ref. [9] (notice that a mistyping in Eq. (103) of [9] has been fixed). In particular, the integral equation that links $\psi_{\text {dist }}$ to the dynamics governed by the BS kernel in the ladder approximation is given by

$$
\begin{aligned}
& \int_{0}^{\infty} \mathrm{d} \gamma^{\prime \prime} \frac{g_{0 L d}^{(+)}\left(\gamma^{\prime \prime}, z\right)}{\left[\gamma+\gamma^{\prime \prime}+z^{2} m^{2}-i \epsilon\right]^{2}} \\
& =\frac{g^{2}}{\mu^{2}} \int_{-\infty}^{\infty} \mathrm{d} \gamma^{\prime \prime} \frac{\theta\left(\gamma^{\prime \prime}\right)}{\left[\gamma+\gamma^{\prime \prime}+z^{2} m^{2}-i \epsilon\right]^{2}}
\end{aligned}
$$




$$
\begin{aligned}
& \times\left\{\theta(z) \theta\left[1-z-\gamma^{\prime \prime} / \mu^{2}\right]+\theta(-z) \theta\left[1+z-\gamma^{\prime \prime} / \mu^{2}\right]\right\} \\
& -\frac{g^{2}}{2(4 \pi)^{2}} \int_{0}^{\infty} \mathrm{d} \gamma^{\prime} \int_{-1}^{1} \mathrm{~d} \zeta^{\prime} g_{0 L d}^{(+)}\left(\gamma^{\prime}, \zeta^{\prime}\right) \int_{-\infty}^{\infty} \mathrm{d} \gamma^{\prime \prime} \\
& \times \frac{1}{\left[\gamma+\gamma^{\prime \prime}+z^{2} m^{2}-i \epsilon\right]^{2}} \theta\left(\gamma^{\prime \prime}\right) \\
& \times\left[\frac{(1+z)}{\left(1+\zeta^{\prime}\right)} \theta\left(\zeta^{\prime}-z\right) h_{0}^{\prime}\left(\gamma^{\prime \prime}, z ; \gamma^{\prime}, \zeta^{\prime}, \mu^{2}\right)\right. \\
& \left.+\frac{(1-z)}{\left(1-\zeta^{\prime}\right)} \theta\left(z-\zeta^{\prime}\right) h_{0}^{\prime}\left(\gamma^{\prime \prime},-z ; \gamma^{\prime},-\zeta^{\prime}, \mu^{2}\right)\right] .
\end{aligned}
$$

Notably, $h_{0}^{\prime}\left(\gamma^{\prime \prime}, z ; \gamma^{\prime}, \zeta^{\prime}, \mu^{2}\right)$ is the proper kernel for a bound state with vanishing energy, as one can check in Ref. [10].

The expression of $h_{0}^{\prime}\left(\gamma^{\prime \prime}, z ; \gamma^{\prime}, \zeta^{\prime}, \mu^{2}\right)$ is given by (see details in Appendix D)

$$
\begin{aligned}
& h_{0}^{\prime}\left(\gamma^{\prime \prime}, z ; \gamma^{\prime}, \zeta^{\prime}, \mu^{2}\right) \\
&=\theta\left.\theta-\mathcal{B}_{0}\left(z, \zeta^{\prime}, \gamma^{\prime}, \gamma^{\prime \prime}, \mu^{2}\right)-2 \mu \sqrt{\zeta^{\prime 2} m^{2}+\gamma^{\prime}}\right] \\
& \quad \times {\left[-\frac{\mathcal{B}_{0}\left(z, \zeta^{\prime}, \gamma^{\prime}, \gamma^{\prime \prime}, \mu^{2}\right)}{\mathcal{A}_{0}\left(\zeta^{\prime}, \gamma^{\prime}\right) \Delta_{0}\left(z, \zeta^{\prime}, \gamma^{\prime}, \gamma^{\prime \prime}, \mu^{2}\right)} \frac{1}{\gamma^{\prime \prime}}\right.} \\
&\left.+\frac{\left(1+\zeta^{\prime}\right)}{(1+z)} \int_{y_{-}}^{y_{+}} \mathrm{d} y \frac{y^{2}}{\left[y^{2} \mathcal{A}_{0}\left(\zeta^{\prime}, \gamma^{\prime}\right)+y\left(\mu^{2}+\gamma^{\prime}\right)+\mu^{2}\right]^{2}}\right] \\
&-\frac{\left(1+\zeta^{\prime}\right)}{(1+z)} \int_{0}^{\infty} \mathrm{d} y \frac{y^{2}}{\left[y^{2} \mathcal{A}_{0}\left(\zeta^{\prime}, \gamma^{\prime}\right)+y\left(\mu^{2}+\gamma^{\prime}\right)+\mu^{2}\right]^{2}},
\end{aligned}
$$

with

$$
\begin{aligned}
& \mathcal{A}_{0}\left(\zeta^{\prime}, \gamma^{\prime}\right)=\zeta^{\prime 2} m^{2}+\gamma^{\prime}=\zeta^{\prime 2} m^{2}+\gamma^{\prime}>0, \\
& \mathcal{B}_{0}\left(z, \zeta^{\prime}, \gamma^{\prime}, \gamma^{\prime \prime}, \mu^{2}\right)=\mu^{2}+\gamma^{\prime}-\gamma^{\prime \prime} \frac{\left(1+\zeta^{\prime}\right)}{(1+z)} \leq 0, \\
& \Delta_{0}^{2}\left(z, \zeta^{\prime}, \gamma^{\prime}, \gamma^{\prime \prime}, \mu^{2}\right)=\mathcal{B}_{0}^{2}\left(z, \zeta^{\prime}, \gamma^{\prime}, \gamma^{\prime \prime}, \mu^{2}\right) \\
& -4 \mu^{2} \mathcal{A}_{0}\left(\zeta^{\prime}, \gamma^{\prime}\right) \geq 0, \\
& y_{ \pm}=\frac{1}{2 \mathcal{A}_{0}\left(\zeta^{\prime}, \gamma^{\prime}\right)} \\
& \quad \times\left[-\mathcal{B}_{0}\left(z, \zeta^{\prime}, \gamma^{\prime}, \gamma^{\prime \prime}, \mu^{2}\right) \pm \Delta_{0}\left(z, \zeta^{\prime}, \gamma^{\prime}, \gamma^{\prime \prime}, \mu^{2}\right)\right] .
\end{aligned}
$$

The zero-energy limit of Eq. (26), i.e. the integral equation based on the uniqueness of the Nakanishi weight function, reads (cf. Ref. $[9,10])$ is

$$
\begin{aligned}
& g_{0 L d}^{(+)}(\gamma, z)=\frac{g^{2}}{\mu^{2}} \theta(\gamma) \theta\left[\mu^{2}(1-|z|)-\gamma\right] \\
& -\frac{g^{2}}{2(4 \pi)^{2}} \theta(\gamma) \int_{-1}^{1} \mathrm{~d} \zeta^{\prime} \int_{0}^{\infty} \mathrm{d} \gamma^{\prime} g_{0 L d}^{(+)}\left(\gamma^{\prime}, \zeta^{\prime}\right) \\
& \quad \times\left[\frac{(1+z)}{\left(1+\zeta^{\prime}\right)} \theta\left(\zeta^{\prime}-z\right) h_{0}^{\prime}\left(\gamma, z ; \gamma^{\prime}, \zeta^{\prime}, \mu^{2}\right)\right. \\
& \left.+\frac{(1-z)}{\left(1-\zeta^{\prime}\right)} \theta\left(z-\zeta^{\prime}\right) h_{0}^{\prime}\left(\gamma,-z ; \gamma^{\prime},-\zeta^{\prime}, \mu^{2}\right)\right] .
\end{aligned}
$$

It should be pointed out that the presence of a non-smooth behavior, like the discontinuity around $\gamma \sim \mu^{2}(1-|z|)$, is expected if one has to reproduce the singular behavior of the distorted part of the scattering wave function [cf. Eqs. (10) and (12)].

As illustrated in Sect. 4, we have taken profit of the general structure of the weight function suggested by Eq. (53) for obtaining numerical solutions of both Eqs. (50) and (53), and eventually calculating the scattering lengths.

It is worth noting that the scattering length given by Eq. (48) represents a normalization for $g_{0 L d}^{(+)}\left(\gamma^{\prime \prime}, \zeta^{\prime}\right)$, when $\mu \leq$ $2 m$. As a matter of fact, from Eq. (53), one realizes that the inhomogeneous term is different from zero only for

$0 \leq \gamma \leq \mu^{2}(1-|z|)$.

Moreover, within the previous interval and $\mu \leq 2 m$, the contribution to the kernel $h_{0}^{\prime}$ that contains

$\theta\left[\gamma \frac{\left(1 \pm \zeta^{\prime}\right)}{(1 \pm z)}-\gamma^{\prime}-\mu^{2}-2 \mu \sqrt{\zeta^{\prime 2} m^{2}+\gamma^{\prime}}\right]$

disappears, since

$$
\begin{aligned}
& \gamma \frac{\left(1 \pm \zeta^{\prime}\right)}{(1 \pm z)}-\gamma^{\prime}-\mu^{2}-2 \mu \sqrt{\zeta^{\prime 2} m^{2}+\gamma^{\prime}} \\
& \quad \leq \mu^{2} \frac{(1-|z|)}{(1 \pm z)}\left(1 \pm \zeta^{\prime}\right)-\mu^{2}-2 \mu m\left|\zeta^{\prime}\right| \\
& \quad<\mu^{2}\left(1 \pm \zeta^{\prime}\right)-\mu^{2}-2 \mu m\left|\zeta^{\prime}\right|<\mu\left|\zeta^{\prime}\right|( \pm \mu-2 m) .
\end{aligned}
$$

The final step in the above expression is always negative when $\mu<2 m$.

Therefore, for $0 \leq \gamma \leq \mu^{2}(1-|z|)$ and $\mu \leq 2 m$, one has

$$
\begin{aligned}
& g_{0 L d}^{(+)}(\gamma, z)=\frac{g^{2}}{\mu^{2}}+\frac{g^{2}}{2(4 \pi)^{2}} \int_{-1}^{1} \mathrm{~d} \zeta^{\prime} \int_{0}^{\infty} \mathrm{d} \gamma^{\prime} \\
& \quad \times \int_{0}^{\infty} \mathrm{d} y \frac{y^{2} g_{0 L d}^{(+)}\left(\gamma^{\prime}, \zeta^{\prime}\right)}{\left[y^{2} \mathcal{A}_{0}\left(\zeta^{\prime}, \gamma^{\prime}\right)+y\left(\mu^{2}+\gamma^{\prime}\right)+\mu^{2}\right]^{2}} \\
& =-16 \pi m a,
\end{aligned}
$$

where Eq. (48) has been exploited in the last step.

\section{Results}

In this section, the numerical studies of both the scattering length and the distorted part of the 3D wave function are presented. First of all, let us illustrate our numerical method for solving the two integral equations in (50) and (53). The main ingredient is the following decomposition of the Nakanishi weight function that takes into account the singular behavior shown in Eq. (53), but also the result in Eq. (55), that holds for $\mu \leq 2 m$ (this is always fulfilled for realistic cases): 
$g_{0 L d}^{(+)}(\gamma, z)=\beta \theta(-t)+\theta(t) \sum_{\ell=0}^{N_{z}} \sum_{j=0}^{N_{g}} A_{\ell j} G_{\ell}(z) \mathcal{L}_{j}(t)$,

where (1) $t=\gamma-\mu^{2}(1-|z|)$, (2) the functions $G_{\ell}(z)$ are given in terms of even Gegenbauer polynomials, $C_{2 \ell}^{(5 / 2)}(z)$ [recall that $g_{0 L d}^{(+)}(\gamma, z)$ must be even in $z$ ] by

$$
\begin{aligned}
G_{\ell}(z)= & 4\left(1-z^{2}\right) \Gamma(5 / 2) \\
& \times \sqrt{\frac{(2 \ell+5 / 2)(2 \ell) !}{\pi \Gamma(2 \ell+5)}} C_{2 \ell}^{(5 / 2)}(z),
\end{aligned}
$$

and (3) the functions $\mathcal{L}_{j}(t)$ are expressed in terms of the Laguerre polynomials, $L_{j}(b t)$, by

$\mathcal{L}_{j}(t)=\sqrt{b} L_{j}(b t) e^{-b t / 2}$.

The following orthonormality conditions are fulfilled:

$$
\begin{aligned}
& \int_{-1}^{1} \mathrm{~d} z G_{\ell}(z) G_{n}(z)=\delta_{\ell n}, \\
& \int_{0}^{\infty} \mathrm{d} t \mathcal{L}_{j}(t) \mathcal{L}_{\ell}(t) \\
& =b \int_{0}^{\infty} \mathrm{d} t e^{-b t} L_{j}(b t) L_{\ell}(b t)=\delta_{j \ell} .
\end{aligned}
$$

In Appendix E some details are given for illustrating how Eq. (53) can be numerically solved by using the previous decomposition. In order to speed up the convergence, in the actual calculations the parameter $b=15.0 / \mathrm{m}^{2}$ has been adopted. The finite-range integrations (as those with respect to the variable $z$ and the variable $\gamma$ when integrated up to $\left.\mu^{2}(1-|z|)\right)$ have been performed using a GaussLegendre quadrature rule. The infinite-range integrations, on the other hand, have been performed adopting a GaussLaguerre quadrature method. Finally, the convergence of the expansion given in Eq. (56) is very rapid, and adopting the values $N_{z}=10$ and $N_{g}=24$ well-converging values have been obtained. All the results presented in this section have been obtained for such a choice. Notice that at the end of the calculation $\beta$ as a result was found to be in agreement with the normalization $-16 \pi a$ shown in Eq. (55).

In Tables 1, 2, and 3, the scattering lengths, Eq. (48), calculated by using the Nakanishi weight function obtained by solving both the integral equation (50), $a_{F V S}$, and the integral equation (53), $a_{U N I}$, are shown for $\mu / m=0.15,0.5,1.0$ and values of the coupling constant $\alpha=g^{2} /\left(16 \pi \mathrm{m}^{2}\right)$, which range from a weak-interaction regime to a strong one. Moreover, for the sake of comparison, the results of Ref. [13], $a_{C K}$, evaluated within a totally different framework, based on a direct calculations of the half-off-shell scattering amplitude taking explicitly into account contributions from the singularities affecting the amplitude itself, are presented in the second column. For reference, also the Born values of the scattering lengths are given in the fifth column. From the
Table 1 Comparison, for $\mu / m=0.15$, between the scattering lengths (see Eq. (48)) evaluated in Ref. [13], $a_{C K}$ (second column), and the ones, $a_{F S V}$ (third column) and $a_{U N I}$ (fourth column), calculated by adopting the Nakanishi weight function obtained from Eqs. (50) and (53), respectively. All the calculations are in the ladder approximation. The first column contains the coupling constant $\alpha=g^{2} /\left(16 \pi \mathrm{m}^{2}\right)$. Finally, the fifth column shows the scattering length in Born approximation, Eq. (49). The scattering lengths are in unit $1 / m$. ('Private communication by J. Carbonell)

\begin{tabular}{lllll}
\hline$\alpha$ & $a_{C K}[13]$ & $a_{F S V}$ & $a_{U N I}$ & $a_{B A}$ \\
\hline 0.01 & -0.460 & -0.459 & -0.459 & -0.444 \\
0.05 & -2.70 & -2.65 & -2.65 & -2.22 \\
0.10 & -6.92 & -6.66 & -6.66 & -4.44 \\
0.20 & -34.6 & -29.9 & -29.9 & -8.89 \\
0.30 & 79.5 & 105.9 & 105.0 & -13.3 \\
0.40 & 27.2 & 28.0 & 28.0 & -17.8 \\
0.50 & $17.7^{*}$ & 17.2 & 17.2 & -22.2 \\
0.60 & 12.8 & 11.8 & 11.8 & -26.7 \\
0.70 & 8.66 & 7.73 & 7.72 & -31.1 \\
0.80 & 3.73 & 3.68 & 3.68 & -35.6 \\
0.90 & -4.57 & -1.13 & -1.13 & -40.0 \\
1.00 & -28.1 & -7.89 & -7.89 & -44.4 \\
1.10 & 900. & -19.4 & -19.4 & -48.9 \\
1.50 & 24.7 & 66.9 & 66.9 & -66.7 \\
2.00 & 17.4 & 23.2 & 23.2 & -88.9 \\
2.50 & 14.4 & 12.3 & 12.2 & -111.0 \\
\hline
\end{tabular}

Table 2 The same as in Table 1, but for the mass of the exchanged scalar $\mu / m=0.5$

\begin{tabular}{lllll}
\hline$\alpha$ & $a_{C K}[13]$ & $a_{F S V}$ & $a_{U N I}$ & $a_{B A}$ \\
\hline 0.01 & -0.0403 & -0.0403 & -0.0403 & -0.04 \\
0.05 & -0.209 & -0.209 & -0.209 & -0.20 \\
0.10 & -0.438 & -0.438 & -0.438 & -0.40 \\
0.20 & -0.971 & -0.971 & -0.971 & -0.80 \\
0.30 & -1.64 & -1.64 & -1.64 & -1.20 \\
0.40 & -2.50 & -2.50 & -2.50 & -1.60 \\
0.50 & -3.66 & -3.66 & -3.66 & -2.00 \\
0.60 & -5.34 & -5.34 & -5.34 & -2.60 \\
0.70 & -7.98 & -7.99 & -7.98 & -2.80 \\
0.80 & -12.8 & -12.8 & -12.8 & -3.20 \\
0.90 & -24.7 & -24.7 & -24.8 & -3.60 \\
1.00 & -103.0 & -103.2 & -103.0 & -4.00 \\
1.10 & 62.0 & 61.9 & 61.8 & -4.40 \\
1.50 & 11.0 & 11.0 & 11.0 & -6.00 \\
2.00 & 6.34 & 6.34 & 6.34 & -8.00 \\
2.50 & 4.54 & 4.53 & 4.53 & -10.00 \\
\hline
\end{tabular}

Tables, one can observe a very good agreement among all the three sets of numerical results, but some comments are in order: (1) the comparison between $a_{F S V}$ and $a_{U N I}$ clearly 
Table 3 The same as in Table 1, but for the mass of the exchanged scalar $\mu / m=1.0$

\begin{tabular}{lcccc}
\hline$\alpha$ & $a_{C K}[13]$ & $a_{F S V}$ & $a_{U N I}$ & $a_{B A}$ \\
\hline 0.01 & -0.010 & -0.010 & -0.010 & -0.01 \\
0.05 & -0.051 & -0.051 & -0.051 & -0.05 \\
0.10 & -0.104 & -0.104 & -0.104 & -0.10 \\
0.20 & -0.217 & -0.217 & -0.217 & -0.20 \\
0.30 & -0.339 & -0.340 & -0.340 & -0.30 \\
0.40 & -0.474 & -0.474 & -0.474 & -0.40 \\
0.50 & -0.621 & -0.621 & -0.621 & -0.50 \\
0.60 & -0.784 & -0.784 & -0.784 & -0.60 \\
0.70 & -0.965 & -0.966 & -0.966 & -0.70 \\
0.80 & -1.17 & -1.17 & -1.17 & -0.80 \\
0.90 & -1.40 & -1.40 & -1.40 & -0.90 \\
1.00 & -1.66 & -1.66 & -1.66 & -1.00 \\
1.10 & -1.95 & -1.96 & -1.96 & -1.10 \\
1.50 & -3.79 & -3.80 & -3.81 & -1.50 \\
2.00 & -11.1 & -11.3 & -11.3 & -2.00 \\
2.50 & 56.8 & 51.7 & 51.6 & -2.50 \\
\hline
\end{tabular}

confirms that the uniqueness of the Nakanishi weight function can be assumed with a very high degree of confidence, as we have quantitatively shown also for the bound-state case [10,11]; (2) differences between $a_{C K}$ [13] and our calculations are present for $\mu=0.15 \mathrm{~m}$ when the value of $\alpha$ approaches a value which corresponds to a bound state of zero energy. In such a case, the scattering length diverges (let us recall that, for the bound-state case, $\alpha$ is obtained as an eigenvalue of the homogeneous integral equation, in the ladder approximation), or there is a change of sign. Indeed, the above mentioned numerical differences do not represent a big issue (nonetheless it will be numerically investigated elsewhere), given the completely different theoretical frameworks adopted in Ref. [13] and in our work, and the wellknown resonance behavior of the scattering length, when a bound state is approaching a zero-energy scattering state. Finally, it is worth noting that the Born approximation $a_{B A}$ represents a quite good approximation only for small $\alpha$ (see also Fig. 1). Summarizing, the results shown in Tables 1, 2, and 3 , together with the calculations for the bound states $[4,10,11]$, are a very strong evidence that the Nakanishi Ansatz, like the one for scattering states in Eq. (1), represents a reliable tool for solving both homogeneous and inhomogeneous BSE's in Minkowski space.

In Fig. 1, the scattering lengths for the above three values of $\mu / m$ are presented as a function of the absolute value of the scattering length in Born approximation $\left|a_{B A}\right|$ (see Eq. (49) and the last columns in Tables 1, 2 and 3). Interestingly, in the same figure, it is also shown the comparison with the corresponding non-relativistic scattering lengths, evaluated through a well-known expression (see e.g. [24,25]), which exactly reproduce the second Born approximation, viz.

$$
m a=\frac{m a_{B A}}{1+\frac{\mu}{2 m}\left(m a_{B A}\right)} .
$$

The chosen range of $\left|a_{B A}\right|$ is $[0,1.5]$, in unit of the inverse mass $m$ (the mass of the interacting scalars). Beyond this interval, the scattering lengths can change the sign, as illustrated by the above Tables. Moreover, since $m a_{B A}=$ $-\alpha m^{2} / \mu^{2}$, after fixing the value of $\mu / m$ one can follow the dependence of the scattering length on the Yukawa coupling constant $g^{2}$. In particular, from Fig. 1, one can see that

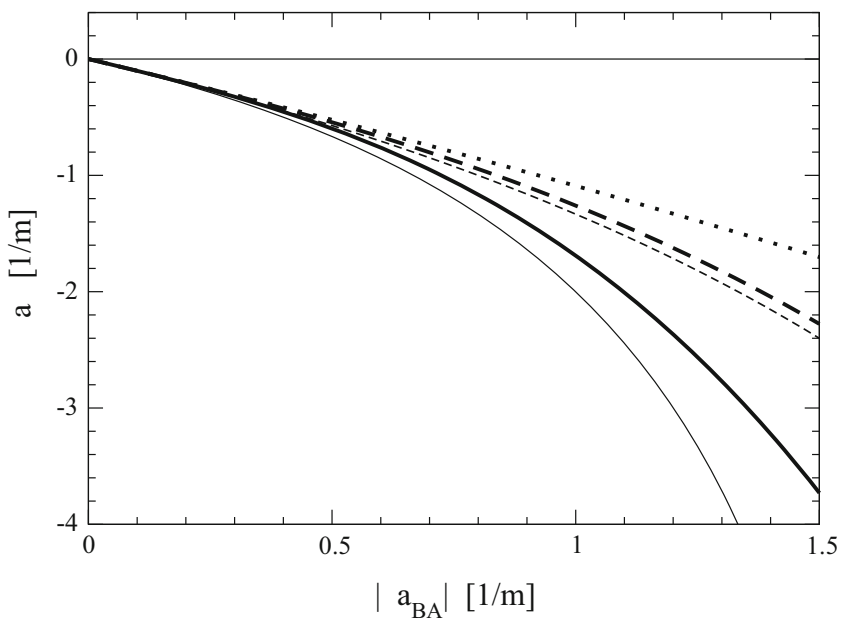

Fig. 1 The scattering lengths, calculated by using Eq. (48), i.e. corresponding to solutions of the inhomogeneous BSE at zero energy, vs. $\left|a_{B A}\right|$ (Eq. (49)). Thick-solid line $a$ for $\mu / m=1$. Thick-dashed line $a$ for $\mu / m=0.5$. Thick-dotted line $a$ for $\mu / m=0.15$. The non-relativistic scattering lengths, represented by the corresponding thin lines, have been calculated by using Eq. (60). Notice that for $\mu / m=0.15$ the nonrelativistic calculation largely overlaps the relativistic one (thick-dotted line), and therefore it is indistinguishable 

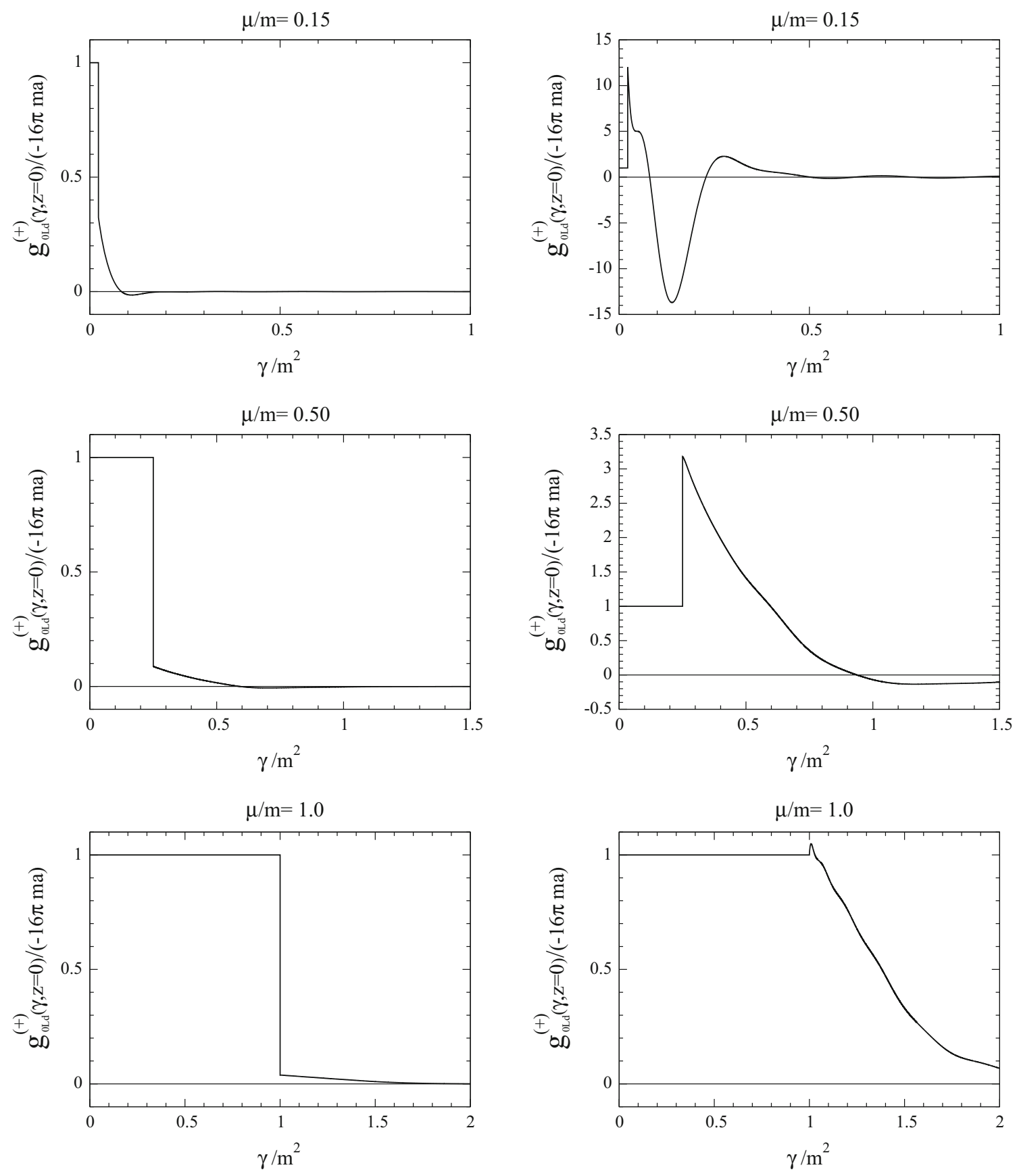

Fig. 2 The Nakanishi weight function $g_{0 L d}^{(+)}(\gamma, z)$, in the zero-energy limit, vs $\gamma / m^{2}$, for $\mu / m=0.15,0.5,1.0$ and $z=0$. Left panels weakinteraction regime with a chosen value $\alpha=0.1$. Right panels strong-interaction regime with a chosen value $\alpha=2.5$

for increasing values of $g^{2}$ and $\mu / m$ the relativistic treatment in Minkowski space, becomes more and more important, as expected, since the effect of the attractive interaction becomes more and more large. Notice that the scalar exchange in Eq. (17) leads to a non-relativistic attractive Yukawa potential. Summarizing, modulo the adopted ladder approximation, the comparison suggests that some care should be taken when one has to consider the effect of the interaction in the description of both hadronic scattering pro- cesses, even in the low-energy regime, and final states, that, e.g., are relevant for hadronic decays.

In Fig. 2, the Nakanishi weight functions for (1) $\mu / m=$ $0.15,0.5,1.0,(2) \alpha=0.1,2.5$, and (3) $z=0$, but running $\gamma / \mathrm{m}^{2}$, are shown. It should be pointed out that, for each value of $\mu / m$, the two values of the coupling constant $\alpha$ are representatives of a weak-interaction regime and a strong one. Moreover, since the Nakanishi weight functions obtained from Eqs. (50) and (53) substantially coincide, only the cal- 

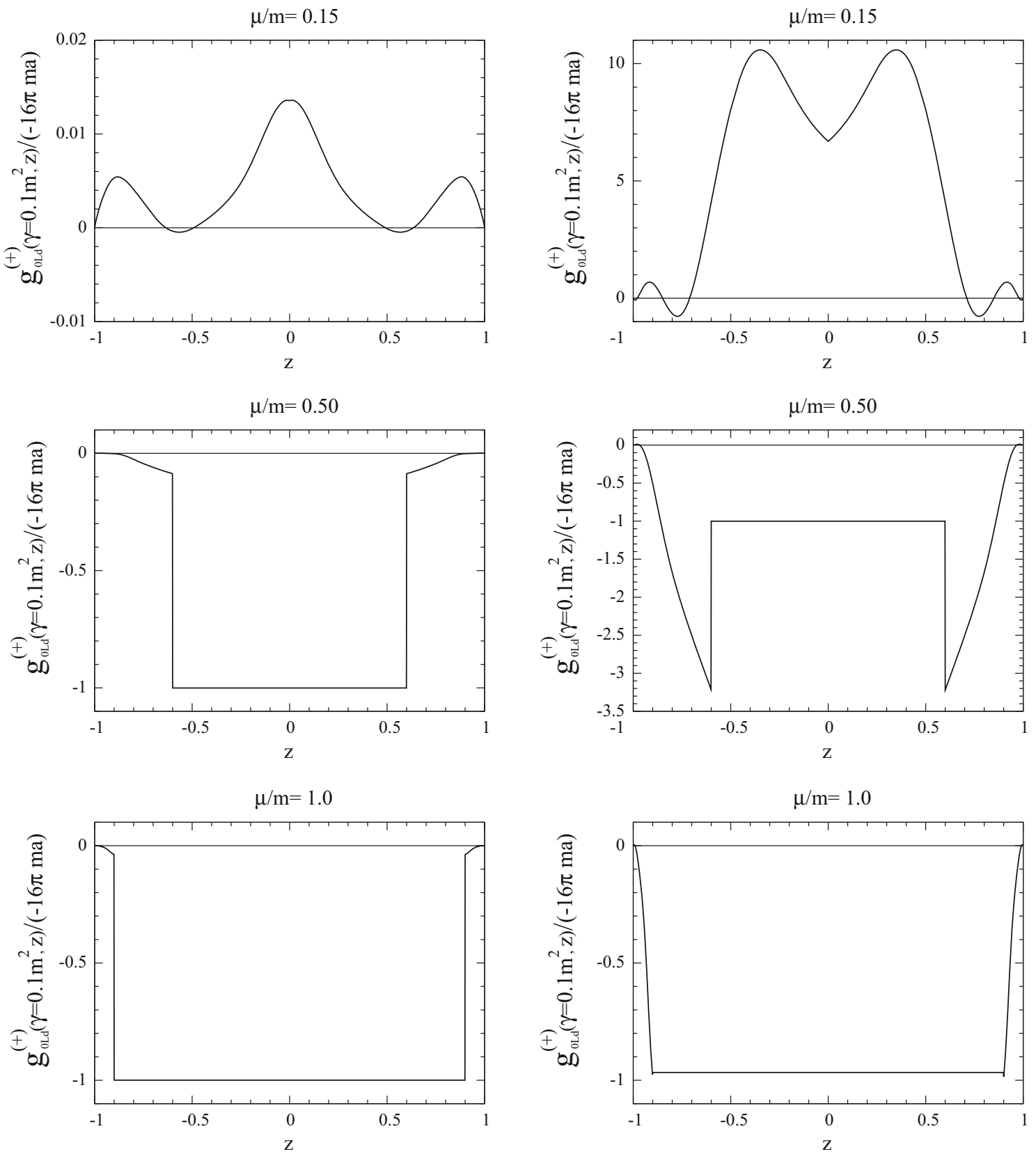

Fig. 3 The same as in Fig. 2 but for running $z$, and $\gamma=0.1 \mathrm{~m}^{2}$

culations corresponding to Eq. (50) are shown. As mentioned at the beginning of this section, the step-function behavior for small $\gamma$ has to be present, and the discontinuities are needed for obtaining the expected singularities in $\psi_{\text {dist }}$, like the one due to the global propagation. In Fig. 2, the transition from the weak regime to the strong one increases the discontinuous behavior, which for large $\mu$ become more and more smooth. Finally, recalling that for a bound state and $\mu \rightarrow 0$, i.e. the Wick-Cutkosky model [20,21], the Nakanishi weight function becomes proportional to $\delta(\gamma)$, it is instructive to see the onset of such a behavior in the upper part of Fig. 2.
For $\gamma=0$ and $|z| \neq 1$, only the first part of the decomposition in Eq. (56), i.e. $g_{0 L d}^{(+)}(\gamma, z) \sim \beta \theta\left(\mu^{2}(1-|z|)\right)$, is dominant, and therefore trivial.

In Fig. 3, the same quantities as in Fig. 2, but for $\gamma / m^{2}=$ 0.1 and running $z$, are also shown. As illustrated by the figure, the Nakanishi weight function acquires a quite discontinuous behavior, as $\mu / m$ increases.

Indeed, it is more profitable to present LF distributions, obtained from the distorted part of the zero-energy 3D scattering wave function. In analogy with the bound-state case (see Refs. [10,11]), one can construct transverse and longi- 
tudinal LF momentum distributions. In particular, one gets the following expression for $\psi_{\mathrm{dist}}^{(L d)}\left(z, \gamma ; \kappa^{2}=z_{i}=0\right)$ :

$$
\begin{aligned}
& \psi_{\text {dist }}^{(L d)}\left(z, \gamma ; \kappa^{2}=z_{i}=0\right) \\
& =\frac{\left(1-z^{2}\right)}{4} \int_{0}^{\infty} \mathrm{d} \gamma^{\prime} \frac{g_{0 L d}^{(+)}\left(\gamma^{\prime}, z\right)}{\left[\gamma^{\prime}+\gamma+z^{2} m^{2}\right]^{2}} .
\end{aligned}
$$

It should be noticed that inserting in Eq. (61) only the first part of the decomposition in Eq. (56), one quickly again obtains the singular behavior due to the global propagation as shown in Eq. (12), viz.

$$
\begin{aligned}
& \psi_{\text {dist }}^{(L d)}\left(z, \gamma ; \kappa^{2}=z_{i}=0\right) \sim \beta \frac{\left(1-z^{2}\right)}{4} \\
& \quad \times\left[\frac{1}{\gamma+z^{2} m^{2}}-\frac{1}{\mu^{2}(1-|z|)+\gamma+z^{2} m^{2}}\right]=\beta \\
& \quad \times \frac{\left(1-z^{2}\right)}{4}\left[\frac{\mu^{2}(1-|z|)}{\left(\gamma+z^{2} m^{2}\right)\left[\mu^{2}(1-|z|)+\gamma+z^{2} m^{2}\right]}\right] .
\end{aligned}
$$

Therefore, one has to expect singularities in the LF momentum distributions, that we would introduce in analogy with the ones for the bound states [10]. Let us emphasize that only for the bound states they have a probabilistic interpretation. One could defines (1) the distorted transverse LF distribution

$$
\begin{aligned}
& \mathcal{P}_{\text {dist }}(\gamma)=\frac{1}{2(2 \pi)^{3}} \int_{0}^{1} \frac{\mathrm{d} \xi}{2 \xi(1-\xi)} \\
& \quad \times \int_{0}^{2 \pi} \mathrm{d} \phi\left[\psi_{\text {dist }}^{(L d)}\left(z, \gamma ; \kappa^{2}=z_{i}=0\right)\right]^{2}=\frac{1}{(16 \pi)^{2}} \\
& \quad \times \int_{-1}^{1} \mathrm{~d} z\left(1-z^{2}\right)\left[\int_{0}^{\infty} \mathrm{d} \gamma^{\prime} \frac{g_{0 L d}^{(+)}\left(\gamma^{\prime}, z\right)}{\left[\gamma^{\prime}+\gamma+z^{2} m^{2}\right]^{2}}\right]^{2}
\end{aligned}
$$

and (2) the longitudinal one, viz.

$$
\begin{aligned}
& \phi_{\text {dist }}(\xi)=\frac{1}{(2 \pi)^{3}} \frac{1}{2 \xi(1-\xi)} \\
& \times \int \mathrm{d} \mathbf{k}_{\perp}\left[\psi_{\text {dist }}^{(L d)}\left(z, \gamma ; \kappa^{2}=z_{i}=0\right)\right]^{2}=2 \frac{\left(1-z^{2}\right)}{(16 \pi)^{2}} \\
& \quad \times \int_{0}^{\infty} \mathrm{d} \gamma\left[\int_{0}^{\infty} \mathrm{d} \gamma^{\prime} \frac{g_{0 L d}^{(+)}\left(\gamma^{\prime}, z\right)}{\left[\gamma^{\prime}+\gamma+z^{2} m^{2}\right]^{2}}\right]^{2}
\end{aligned}
$$

with the fraction of longitudinal momentum given by

$$
\xi=\frac{1-z}{2}=\frac{1}{p^{+}}\left(\frac{p^{+}}{2}+k^{+}\right) .
$$

For the sake of presentation, it is useful to partially removing the singularities affecting the above distributions. Therefore, in Fig. $4, \gamma^{2} \mathcal{P}(\gamma)$ and $|1-2 \xi|^{3 / 2} \phi(\xi)$ are shown. Figure 4 illustrates the overall behavior of the LF distributions by varying the coupling $\alpha$ and the mass of the exchanged scalar $\mu$, as in Figs. 2 and 3. It is worth noting the order-of-magnitude differences, when the coupling $\alpha$ is changed, but the typical features that one expects are still recognizable. A part the divergent behavior, already pointed out, that can be ascribed to the global propagation, the transverse distribution shows the ultraviolet tail produced by the dominance of a single exchanged scalar, exactly as in the case of the corresponding distribution for bound states (see Ref. [11]). As to the longitudinal distributions, the expected peak at $\xi=1 / 2$ or $z=0$ is also seen.

The practical use of $\psi_{\text {dist }}$ is given by the evaluation of reactions that need a reliable treatment of the relativistic effects, i.e. when the coupling constant becomes larger and larger.

\section{Conclusions}

In the present paper, our approach [9-11] based on the Nakanishi integral representation of the Bethe-Salpeter amplitude is extended for the first time to the quantitative investigation of the zero-energy limit of the inhomogeneous BetheSalpeter equation, in the ladder approximation, for an interacting system composed of two massive scalars that exchange a massive scalar. This achievement represents a non-trivial task that has allowed us to gain a sound confidence in the Nakanishi Ansatz, as an effective and workable tool for obtaining actual solutions of the homogeneous and inhomogeneous BSE's in Minkowski space. Indeed, the same approach that leads to a careful description of the bound states also yields a very accurate evaluation of the scattering length, as shown in Tables 1, 2, and 3, by the quantitative comparisons with the same observable evaluated within a totally different framework, based on the direct calculation of the contributions from the singularities of the inhomogeneous BSE [13,14].

As in the bound-state case, we have performed the calculations by using the integral representation of the BS amplitude in terms of the Nakanishi weight function, Eq. (1), which explicitly shows the analytic dependence of the BS amplitude upon the invariant kinematical scalars of the scattering process under scrutiny. Then, by applying the LF projection onto the null-plane to the inhomogeneous BSE in Minkowski space, Eq. (4) (without self-energy and vertex corrections), one is able to formally obtain an inhomogeneous integral equation for the Nakanishi weight function that depends upon real variables. Its expression in the ladder approximation is given by Eq. (18). One can deduce another inhomogeneous integral equation for the Nakanishi weight function, Eq. (26), by assuming the uniqueness of the Nakanishi weight function to be valid, also in the nonperturbative regime (recall that the theorem was demonstrated by Nakanishi [12] in a perturbative framework, but taking into account the whole infinite set of diagrams contributing to a given $n$-leg amplitude).

The numerical comparisons for the scattering lengths, obtained by using our Eqs (50) and (53), and the corre- 
$\mu / \mathrm{m}=0.15$

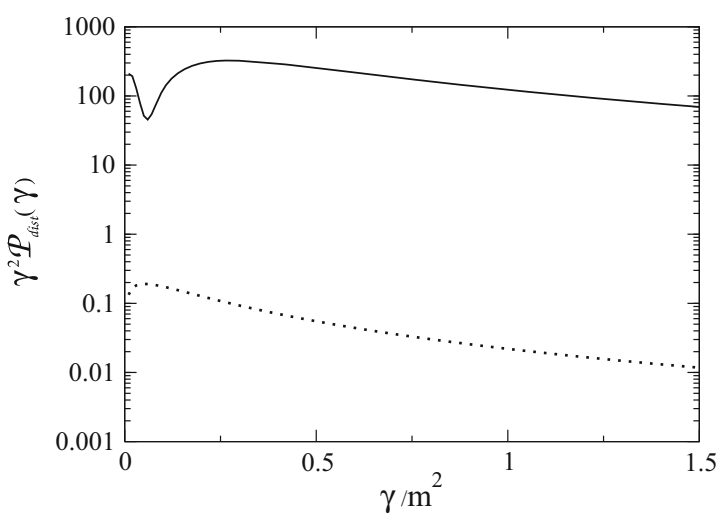

$\mu / \mathrm{m}=0.50$

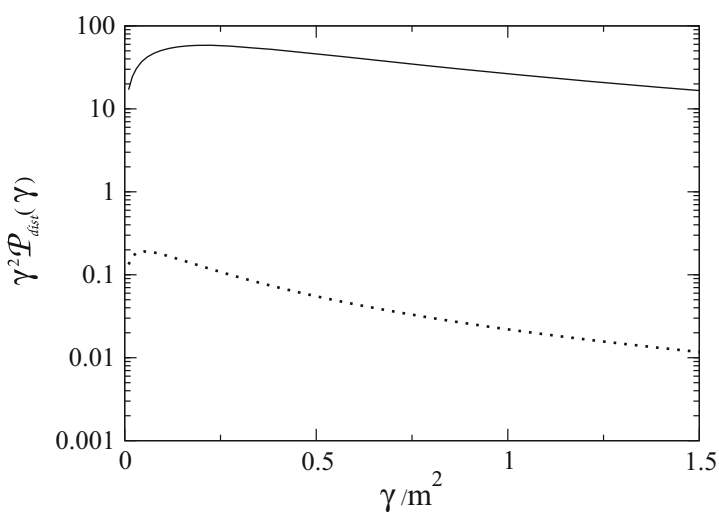

$\mu / \mathrm{m}=1.0$

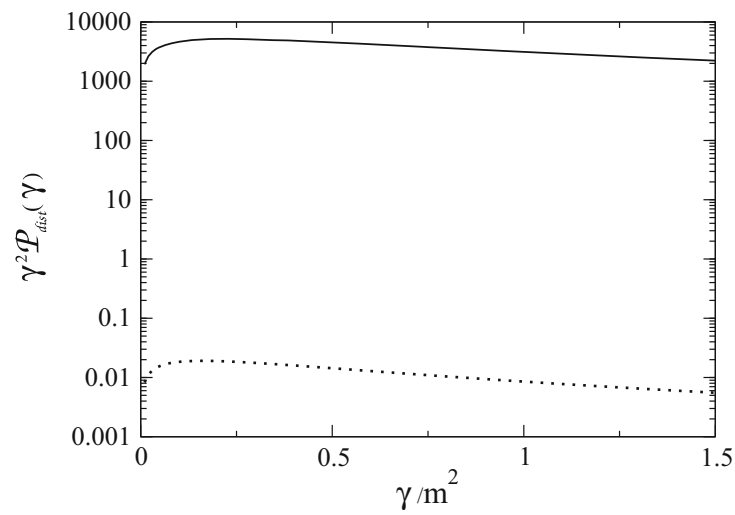

Fig. 4 The LF distributions obtained from the distorted part of the 3D LF scattering wave function (see Eq. (61) for $\mu / m=0.15,0.5,1.0$ and zero-energy limit. Left panels transverse LF-distribution $\gamma^{2} \mathcal{P}(\gamma)$ vs. the $\gamma / m^{2}$ (cf. the transverse LF-distribution expression in Eq.(63)).

sponding quantities calculated in Ref. [13] are shown in great detail in Tables 1, 2, and 3. It must be emphasized that the high accuracy reached by our calculations is due to the new decomposition in Eq. (56), suitable for obtaining the numerical solutions of the two inhomogeneous integral equations, involving the Nakanishi weight function. The comparison with the non-relativistic scattering lengths (cf. Fig. 1) has $\mu / \mathrm{m}=0.15$

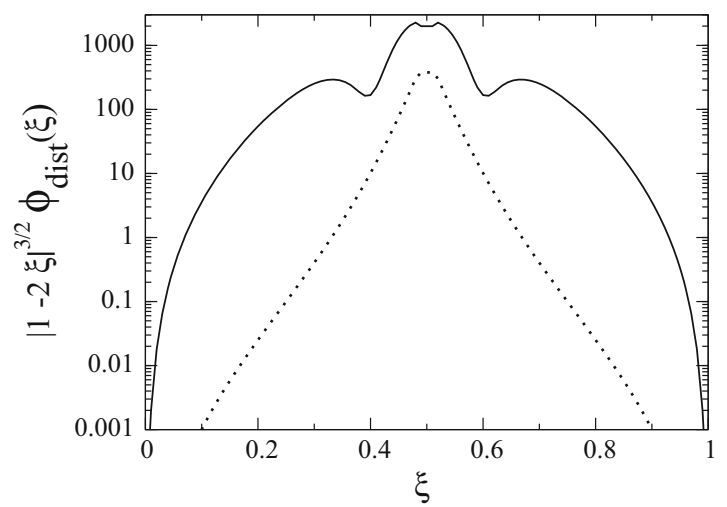

$\mu / \mathrm{m}=0.50$

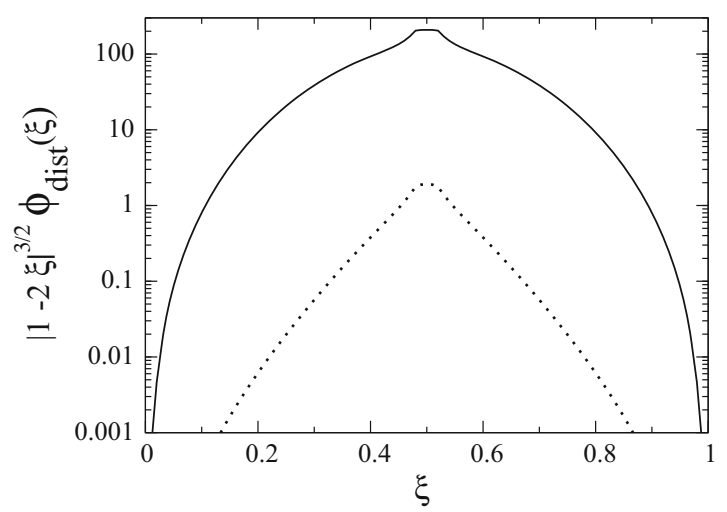

$\mu / \mathrm{m}=1.0$

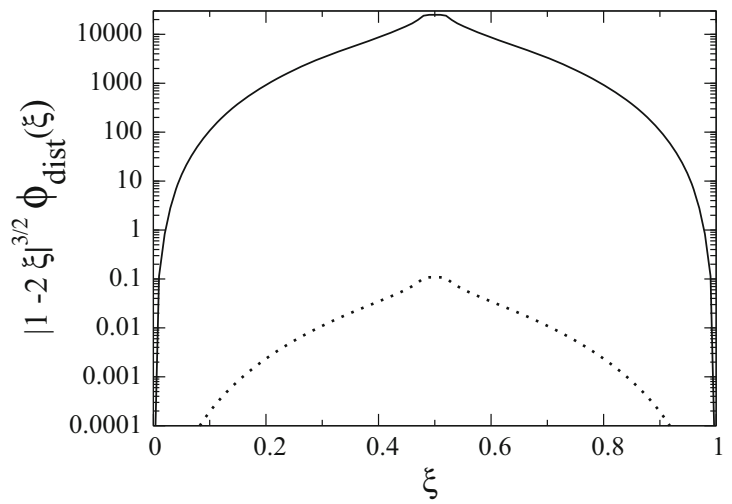

Solid line strong-interaction regime with $\alpha=2.5$; dotted line weakinteraction regime with $\alpha=0.1$. Right panels the same as in the left panel, but for $|1-2 \xi|^{3 / 2} \phi(\xi)$ (cf. the longitudinal LF-distribution in Eq. (64))

illustrated the potential impact of a proper treatment of the relativistic effects on the investigation of hadronic scattering states, even in the low-energy regime. Indeed, before drawing definite conclusions, it is desirable to improve our approach by considering quite relevant issues, like the ones originating from higher order corrections in the coupling. Therefore, within the Nakanishi framework one should also address rel- 
ativistic effects from vertex corrections, self-energy contributions, as well as a richer content in the interaction kernel (e.g. by including the cross-ladder diagram as in Ref. [5]).

For the sake of completeness, the behavior of the Nakanishi weight functions, in the zero-energy limit, for weak- and strong-interaction regimes have been shown in Figs. 2 and 3. Those figures illustrate the non-smooth behavior of the Nakanishi weight functions for certain ranges of the variables, which is an inheritance of the singular behavior of the scattering states. Finally, we have defined longitudinal and transverse LF momentum distributions, in analogy with the bound-state case (but without the probabilistic interpretation, entailed by the normalization of a bound state). Those distributions are shown in Fig. 4, for the sake of illustration and reference purpose. It should be noticed that the transverse LF distributions show the expected power-like ultraviolet behavior, already found in the bound-state case.

In conclusion, the Nakanishi Ansatz for the BS amplitude allows one to numerically solve in a very accurate way the inhomogeneous BSE, at least in the zero-energy limit. Such an outcome of our approach, together with the very nice results obtained for the bound-state case, strongly encourages one to move to positive-energy scattering states, in order to evaluate the phase shifts. If the phase shifts evaluated within our approach (presented elsewhere [18]) agree with the ones in the literature [13], then the reliability of the Nakanishi Ansatz as a starting guess for obtaining exact solutions of BSE's in Minkowski space could make a substantial step forward, confirming the great potential of this method, which can be applied to many other cases, with different dimensions [26], statistics, kernels, etc.

Acknowledgments We gratefully thank Jaume Carbonell and Vladimir Karmanov for very stimulating discussions. TF acknowledges the warm hospitality of INFN Sezione di Pisa and thanks the partial financial support from the Conselho Nacional de Desenvolvimento Científico e Tecnológico (CNPq), the Fundação de Amparo à Pesquisa do Estado de São Paulo (FAPESP). GS thanks the partial support of Coordenação de Aperfeiçoamento de Pessoal de Nível Superior (CAPES) of Brazil. MV and GS acknowledge the warm hospitality of the Instituto Tecnológico de Aeronáutica, São José dos Campos, São Paulo, Brazil, where part of this work was performed.

Open Access This article is distributed under the terms of the Creative Commons Attribution 4.0 International License (http://creativecomm ons.org/licenses/by/4.0/), which permits unrestricted use, distribution, and reproduction in any medium, provided you give appropriate credit to the original author(s) and the source, provide a link to the Creative Commons license, and indicate if changes were made. Funded by SCOAP ${ }^{3}$.

\section{Appendix A: Boundary properties of the Nakanishi weight function $g^{(+)}\left(\gamma^{\prime}, z^{\prime}, z^{\prime \prime}\right)$}

This appendix is devoted to the analysis of the relation between (1) the Nakanishi weight function $g^{(+)}\left(\gamma^{\prime}, z^{\prime}, z^{\prime \prime}\right)$, which yields the integral representation of the distorted part of the 3D LF scattering wave function, and (2) the weight function $\widetilde{\mathcal{G}}^{+}\left(\gamma^{\prime \prime}, \zeta, \zeta^{\prime}\right)$, which yields the integral representation of the half-off-shell T-matrix (cf. Eq. (58) in Ref. [9]). Notice that the dependence upon $\kappa^{2}$ and $z_{i}$ has been dropped for the sake of a light notation. This analysis allows one to obtain the conditions fulfilled by $g^{(+)}\left(\gamma^{\prime}, z^{\prime}, z^{\prime \prime}\right)$ when $z^{\prime}= \pm 1$ and $z^{\prime \prime}= \pm 1$.

It is worth noting that, while for $\widetilde{\mathcal{G}}^{+}\left(\gamma^{\prime \prime}, \zeta, \zeta^{\prime}\right)$ the constraint $\theta\left(1-|\zeta|-\left|\zeta^{\prime}\right|\right)$ holds, for the variables $z$ and $z^{\prime}$ an analogous relation does not exist.

The above mentioned relation between $g^{(+)}\left(\gamma^{\prime}, z^{\prime}, z^{\prime \prime}\right)$ and $\widetilde{\mathcal{G}}^{+}\left(\gamma^{\prime \prime}, \zeta, \zeta^{\prime}\right)$ reads as follows (cf. Eq. (63) in Ref. [9], where a factor of 2 is missing, as well as in Eq. (60), but it was not relevant for the formal discussion, since it can be reabsorbed in $\widetilde{\mathcal{G}}^{+}$):

$$
\begin{aligned}
& g^{(+)}\left(\gamma^{\prime}, z^{\prime}, z^{\prime \prime}\right)=i 2 \int_{0}^{1} \mathrm{~d} \alpha_{1} \int_{0}^{1} \mathrm{~d} \alpha_{2} \frac{1}{\left(1-\alpha_{1}-\alpha_{2}\right)^{3}} \\
& \quad \times \theta\left(1-\alpha_{1}-\alpha_{2}\right) \theta\left(1-\alpha_{1}-\alpha_{2}-\left|z^{\prime}\right|-\left|z^{\prime \prime}-\alpha_{1}+\alpha_{2}\right|\right) \\
& \quad \times \widetilde{\mathcal{G}}^{+}\left(\frac{\gamma^{\prime}}{\left(1-\alpha_{1}-\alpha_{2}\right)}, \frac{z^{\prime}}{\left(1-\alpha_{1}-\alpha_{2}\right)}, \frac{z^{\prime \prime}-\alpha_{1}+\alpha_{2}}{\left(1-\alpha_{1}-\alpha_{2}\right)}\right),
\end{aligned}
$$

where

$z^{\prime}=\zeta\left(1-\alpha_{1}-\alpha_{2}\right)$

$z^{\prime \prime}=\zeta^{\prime}\left(1-\alpha_{1}-\alpha_{2}\right)+\alpha_{1}-\alpha_{2}$.

Notice that the constraints $\theta\left(1-\alpha_{1}-\alpha_{2}\right)$ and $\theta(1-|\zeta|-$ $\left.\left|\zeta^{\prime}\right|\right)=\theta\left(1-\alpha_{1}-\alpha_{2}-\left|z^{\prime}\right|-\left|z^{\prime \prime}-\alpha_{1}+\alpha_{2}\right|\right)$ have been explicitly written, differently from Eq. (58) in Ref. [9].

In what follows it will be shown that the above theta functions lead to a vanishing Nakanishi weight function at $\left|z^{\prime}\right|=1$ or $\left|z^{\prime \prime}\right|=1$.

Given the presence of $\theta\left(1-\alpha_{1}-\alpha_{2}-\left|z^{\prime}\right|-\left|z^{\prime \prime}-\alpha_{1}+\alpha_{2}\right|\right)$ and $0 \leq \alpha_{i} \leq 1$, it is easily seen that for $\left|z^{\prime}\right|=1$ one has

$g^{(+)}\left(\gamma^{\prime}, z^{\prime}= \pm 1, z^{\prime \prime}\right)=0$.

The same holds for $\left|z^{\prime \prime}\right|=1$. First of all, let us perform a change of variables, viz.

$\xi=1-\left(\alpha_{1}+\alpha_{2}\right), \quad \Delta=\alpha_{1}-\alpha_{2}$

$\alpha_{1}=\frac{1-\xi+\Delta}{2}, \quad \alpha_{2}=\frac{1-\xi-\Delta}{2}$,

then Eq. (A.1) becomes

$$
\begin{aligned}
& g^{(+)}\left(\gamma^{\prime}, z^{\prime}, z^{\prime \prime}\right)=i 2 \frac{1}{2} \int_{0}^{1} \mathrm{~d} \xi \\
& \quad \times \int_{-1}^{1} \mathrm{~d} \Delta \frac{\theta(1-\xi+\Delta) \theta(1-\xi-\Delta)}{\xi^{3}} \\
& \quad \times \widetilde{\mathcal{G}}^{+}\left(\frac{\gamma^{\prime}}{\xi}, \frac{z^{\prime}}{\xi}, \frac{z^{\prime \prime}-\Delta}{\xi}\right) \theta\left(\xi-\left|z^{\prime}\right|-\left|z^{\prime \prime}-\Delta\right|\right) .
\end{aligned}
$$


For $z^{\prime \prime}=1$, one gets $z^{\prime \prime}-\Delta \geq 0$, since $\Delta \in[-1,1]$. Then

$$
\begin{aligned}
& \theta(1-\xi-|\Delta|) \theta\left(\xi-\left|z^{\prime}\right|-1\right. \\
& \quad+\Delta)=\theta(1-\xi-|\Delta|) \theta\left[\Delta-(1-\xi)-\left|z^{\prime}\right|\right]=0,
\end{aligned}
$$

since $\Delta \geq(1-\xi)+\left|z^{\prime}\right| \geq 0$ and $1-\xi \geq|\Delta|$. For $z^{\prime \prime}=-1$, one has $z^{\prime \prime}-\Delta \leq 0$, and then

$$
\begin{aligned}
& \theta(1-\xi-|\Delta|) \theta\left[\xi-\left|z^{\prime}\right|\right. \\
& \quad-(1+\Delta)]=\theta(1-\xi-|\Delta|) \theta\left[-(1-\xi)-\left|z^{\prime}\right|-\Delta\right]=0,
\end{aligned}
$$

since $-\Delta \geq(1-\xi)+\left|z^{\prime}\right| \geq 0$ and $1-\xi \geq|\Delta|$. Therefore, from the above results, one gets

$g^{(+)}\left(\gamma^{\prime}, z^{\prime}, z^{\prime \prime}= \pm 1\right)=0$.

\section{Appendix B: The distorted part of the 3D LF scattering wave function}

In this appendix, it will be shown how the expected global free propagation of the constituents can be factorized out in the expression of $\psi_{\text {dist }}^{(+)}$, as in the non-relativistic case. This result is relevant in two respects. On one side, it emphasizes the analogy with the non-relativistic approach, and on the other side it allows one to understand the support of the Nakanishi weight function $g^{(+)}\left(\gamma^{\prime}, z^{\prime}, z ; \gamma_{i}, z_{i}\right)$, when $\gamma^{\prime}$ runs.

In the $\mathrm{CM}$ frame $\left(\mathbf{p}_{\perp}=0\right.$ and $\left.p^{ \pm}=M\right)$, assuming without loss of generality a head-on scattering, i.e. $\gamma_{i}=0$, the 3D LF scattering wave function, is given by [9]

$$
\begin{gathered}
\psi^{(+)}\left(z, \gamma ; \kappa^{2}, z_{i}\right)=p^{+} \frac{\left(1-z^{2}\right)}{4} \int \frac{\mathrm{d} k^{-}}{2 \pi} \Phi^{(+)}(k, p) \\
=p^{+} \frac{\left(1-z^{2}\right)}{4}(2 \pi)^{3} \delta^{(3)}\left(\tilde{k}-\tilde{k}_{i}\right)+\psi_{\text {dist }}\left(z, \gamma ; \kappa^{2}, z_{i}\right),
\end{gathered}
$$

where $\tilde{k} \equiv\left\{k^{+}, \mathbf{k}_{\perp}\right\}$ and $\psi_{\text {dist }}$ is

$$
\begin{aligned}
& \psi_{\text {dist }}\left(z, \gamma ; \kappa^{2}, z_{i}\right)=\frac{\left(1-z^{2}\right)}{4} \int_{-1}^{1} \mathrm{~d} z^{\prime} \int_{-\infty}^{\infty} \mathrm{d} \gamma^{\prime} \\
& \frac{g^{(+)}\left(\gamma^{\prime}, z^{\prime}, z ; \kappa^{2}, z_{i}\right)}{\left[\gamma^{\prime}+\gamma+z^{2} m^{2}+\left(1-z^{2}\right) \kappa^{2}+\frac{M^{2}}{2} z z^{\prime} z_{i}-i \epsilon\right]^{2}} .
\end{aligned}
$$

By using the Nakanishi weight function for the half-off-shell $T$-matrix, one gets the following expression [9]:

$$
\begin{aligned}
& \psi_{\text {dist }}\left(z, \gamma ; \kappa^{2}, z_{i}\right) \\
& =p^{+} \frac{\left(1-z^{2}\right)}{4} \int \frac{\mathrm{d} k^{-}}{2 \pi}\left\langle k^{\mu}\left|G_{0}(p) T(p)\right| k_{i}^{\mu}\right\rangle \\
& =p^{+} \frac{\left(1-z^{2}\right)}{4} \int \frac{\mathrm{d} k^{-}}{2 \pi} \frac{i}{\left(\frac{p}{2}+k\right)^{2}-m^{2}+i \epsilon}
\end{aligned}
$$

$$
\begin{aligned}
& \times \frac{i}{\left(\frac{p}{2}-k\right)^{2}-m^{2}+i \epsilon} \int_{-1}^{1} \mathrm{~d} z^{\prime} \int_{-1}^{1} \mathrm{~d} \zeta^{\prime} \int_{-\infty}^{\infty} \mathrm{d} \gamma^{\prime} \\
& \times \frac{\widetilde{\mathcal{G}}^{+}\left(\gamma^{\prime}, z^{\prime}, \zeta^{\prime} ; \kappa^{2}, z_{i}\right) \theta\left(1-\left|z^{\prime}\right|-\left|\zeta^{\prime}\right|\right)}{k^{2}+\frac{p^{2}}{4}-m^{2}+\zeta^{\prime} p \cdot k+z^{\prime} 2 k \cdot k_{i}-\gamma^{\prime}+i \epsilon} .
\end{aligned}
$$

Then one can write

$$
\begin{aligned}
\psi_{\text {dist }}\left(z, \gamma ; \kappa^{2}, z_{i}\right) & =p^{+} \frac{\left(1-z^{2}\right)}{4} \int_{-1}^{1} \mathrm{~d} z^{\prime} \int_{-1}^{1} \mathrm{~d} \zeta^{\prime} \int_{-\infty}^{\infty} \mathrm{d} \gamma^{\prime} \\
= & \times \widetilde{\mathcal{G}}^{+}\left(\gamma^{\prime}, z^{\prime}, \zeta^{\prime} ; \kappa^{2}, z_{i}\right) \theta\left(1-\left|z^{\prime}\right|-\left|\zeta^{\prime}\right|\right) \int \frac{\mathrm{d} k^{-}}{2 \pi} \\
& \times \frac{1}{\left(M / 2+k^{+}\right)\left(M / 2-k^{+}\right)} \frac{1}{\left(k^{+}+\zeta^{\prime} \frac{M}{2}+z^{\prime} k_{i}^{+}\right)} \\
& \times \frac{1}{\left(\frac{p}{2}+k\right)^{-}-\left(\frac{p}{2}+k\right)_{o n}^{-}+i \epsilon /\left(M / 2+k^{+}\right)} \\
& \times \frac{1}{\left(\frac{p}{2}-k\right)^{-}-\left(\frac{p}{2}-k\right)_{o n}^{-}+i \epsilon /\left(M / 2-k^{+}\right)} \\
& \times \frac{1}{k^{-}+\frac{k^{+}\left(\zeta^{\prime} \frac{M}{2}+z^{\prime} k_{i}^{-}\right)-\gamma-\gamma^{\prime}-\kappa^{2}+i \epsilon}{\left(k^{+}+\zeta^{\prime} \frac{M}{2}+z^{\prime} k_{i}^{+}\right)}}
\end{aligned}
$$

where

$$
\begin{aligned}
& \left(\frac{p}{2}+k\right)_{o n}^{-}=\frac{2\left(m^{2}+\gamma\right)}{M(1-z)}, \\
& \left(\frac{p}{2}-k\right)_{o n}^{-}=\frac{2\left(m^{2}+\gamma\right)}{M(1+z)},
\end{aligned}
$$

with $k^{+}=-z M / 2$. Since $k_{i}^{+}=-k_{i}^{-}=-z_{i} M / 2$, one gets

$$
\begin{aligned}
& \psi_{\text {dist }}\left(z, \gamma ; \kappa^{2}, z_{i}\right)=-\frac{2}{M^{2}} \int_{-1}^{1} \mathrm{~d} z^{\prime} \int_{-1}^{1} \mathrm{~d} \zeta^{\prime} \int_{-\infty}^{\infty} \mathrm{d} \gamma^{\prime} \\
& \times \widetilde{\mathcal{G}}^{+}\left(\gamma^{\prime}, z^{\prime}, \zeta^{\prime} ; \kappa^{2}, z_{i}\right) \frac{\theta\left(1-\left|z^{\prime}\right|-\left|\zeta^{\prime}\right|\right)}{\left(\zeta^{\prime}-z-z^{\prime} z_{i}\right)} \\
& \times \int \frac{\mathrm{d} k^{-}}{2 \pi} \frac{1}{\frac{M}{2}+k^{-}-\left(\frac{p}{2}+k\right)_{o n}^{-}+i 2 \epsilon /[M /(1-z)]} \\
& \times \frac{1}{\frac{M}{2}-k^{-}-\left(\frac{p}{2}-k\right)_{o n}^{-}+i 2 \epsilon /[M(1+z)]} \\
& \times \frac{1}{\left[k^{-}-k_{N a}^{-}+i 2 \epsilon /\left[M\left(\zeta^{\prime}-z-z^{\prime} z_{i}\right)\right]\right.},
\end{aligned}
$$

with

$$
\begin{aligned}
k_{N a}^{-}= & \frac{2}{M\left(\zeta^{\prime}-z-z^{\prime} z_{i}\right)} \\
& \times\left[\frac{M^{2}}{4} z\left(\zeta^{\prime}+z^{\prime} z_{i}\right)+\gamma+\gamma^{\prime}+\kappa^{2}\right] .
\end{aligned}
$$

One has the following poles (recall that $1>z>-1$ ):

$$
\begin{aligned}
& k_{L}=\left(\frac{p}{2}+k\right)_{\text {on }}^{-}-\frac{M}{2}-i \epsilon, \\
& k_{U}=-\left(\frac{p}{2}-k\right)_{\text {on }}^{-}+\frac{M}{2}+i \epsilon,
\end{aligned}
$$


$k_{L U}=k_{N a}^{-}-i \frac{2 \epsilon}{M\left(\zeta^{\prime}-z-z^{\prime} z_{i}\right)}$.

In order to evaluate the analytic integration on $k^{-}$, one can consider the following two cases.

If $\zeta^{\prime}>z+z^{\prime} z_{i}$, one can close the integration contour into the upper plane, taking the residue at $k_{U}$, i.e.

$$
\begin{aligned}
\int_{-\infty}^{\infty} \frac{\mathrm{d} k^{-}}{2 \pi} \frac{1}{\left[k^{-}-k_{L}\right]} \frac{1}{\left[-k^{-}+k_{U}\right]} \frac{1}{\left[k^{-}-k_{L U}\right]} \\
=-i \frac{1}{\left[M-\left(\frac{p}{2}-k\right)_{o n}^{-}-\left(\frac{p}{2}+k\right)_{o n}^{-}+i \epsilon\right]} \\
\quad \times \frac{1}{\left[\frac{M}{2}-\left(\frac{p}{2}-k\right)_{o n}^{-}-k_{N a}^{-}+i \epsilon\right]} \\
=-i \frac{M^{2}}{8}\left(\zeta^{\prime}-z-z^{\prime} z_{i}\right) \\
\quad \times \frac{\left(1-z^{2}\right)}{\left[\kappa^{2}\left(1-z^{2}\right)+m^{2} z^{2}+\gamma-i \epsilon\right]} \frac{(1+z)}{\left(1+\zeta^{\prime}-z^{\prime} z_{i}\right)} \\
\quad \times \frac{1}{\kappa^{2}\left(1-z^{2}\right)+m^{2} z^{2}+\gamma+\frac{(1+z)\left(\frac{M^{2}}{2} z z^{\prime} z_{i}+\gamma^{\prime}\right)}{\left(1+\zeta^{\prime}-z^{\prime} z_{i}\right)}-i \epsilon} .
\end{aligned}
$$

If $z+z^{\prime} z_{i}>\zeta^{\prime}$, one can close the integration contour into the lower plane, taking the residue at $k_{L}$, i.e.

$$
\begin{aligned}
\int_{-\infty}^{\infty} & \frac{\mathrm{d} k^{-}}{2 \pi} \frac{1}{\left[k^{-}-k_{L}\right]} \frac{1}{\left[-k^{-}+k_{U}\right]} \frac{1}{\left[k^{-}-k_{L U}\right]} \\
= & -i \frac{M^{2}}{8}\left(\zeta^{\prime}-z-z^{\prime} z_{i}\right) \\
& \times \frac{(1-z)}{\left(1-\zeta^{\prime}+z^{\prime} z_{i}\right)} \frac{\left(1-z^{2}\right)}{\left[\kappa^{2}\left(1-z^{2}\right)+m^{2} z^{2}+\gamma-i \epsilon\right]} \\
& \times \frac{1}{\kappa^{2}\left(1-z^{2}\right)+m^{2} z^{2}+\gamma+\frac{(1-z)\left(\frac{M^{2}}{2} z z^{\prime} z_{i}+\gamma^{\prime}\right)}{\left(1-\zeta^{\prime}+z^{\prime} z_{i}\right)}-i \epsilon},
\end{aligned}
$$

where $\left(\zeta^{\prime}-z-z^{\prime} z_{i}\right) \epsilon \rightarrow-\epsilon$, since $\left(\zeta^{\prime}-z-z^{\prime} z_{i}\right)<0$.

Collecting all the above results, one gets the following expression for $\psi_{\text {dist }}$ :

$$
\begin{aligned}
& \psi_{\text {dist }}\left(z, \gamma ; \kappa^{2}, z_{i}\right) \\
& =i \frac{\left(1-z^{2}\right)}{4} \frac{1}{\left[\kappa^{2}\left(1-z^{2}\right)+m^{2} z^{2}+\gamma-i \epsilon\right]} \\
& \quad \int_{-1}^{1} \mathrm{~d} z^{\prime} \int_{-1}^{1} \mathrm{~d} \zeta^{\prime} \int_{-\infty}^{\infty} \mathrm{d} \gamma^{\prime} \widetilde{\mathcal{G}}^{+}\left(\gamma^{\prime}, z^{\prime}, \zeta^{\prime} ; \kappa^{2}, z_{i}\right) \\
& \quad \times\left[\frac{(1+z)}{\left(1+\zeta^{\prime}-z^{\prime} z_{i}\right)}\right. \\
& \quad \times \frac{\theta\left(\zeta^{\prime}-z-z^{\prime} z_{i}\right) \theta\left(1-\left|z^{\prime}\right|-\left|\zeta^{\prime}\right|\right)}{\kappa^{2}\left(1-z^{2}\right)+m^{2} z^{2}+\gamma+\frac{(1+z)\left(\frac{M^{2}}{2} z z^{\prime} z_{i}+\gamma^{\prime}\right)}{\left(1+\zeta^{\prime}-z^{\prime} z_{i}\right)}-i \epsilon}
\end{aligned}
$$

$$
\begin{aligned}
& +\frac{(1-z)}{\left(1-\zeta^{\prime}+z^{\prime} z_{i}\right)} \\
& \left.\times \frac{\theta\left(z+z_{i} z^{\prime}-\zeta^{\prime}\right) \theta\left(1-\left|z^{\prime}\right|-\left|\zeta^{\prime}\right|\right)}{\kappa^{2}\left(1-z^{2}\right)+m^{2} z^{2}+\gamma+\frac{(1-z)\left(\frac{M^{2}}{2} z z^{\prime} z_{i}+\gamma^{\prime}\right)}{\left(1-\zeta^{\prime}+z^{\prime} z_{i}\right)}-i \epsilon}\right] .
\end{aligned}
$$

One can again obtain the expression in Eq. (B.6) by applying the Feynman trick to Eq. (B.15). For instance, one has

$$
\begin{aligned}
& \frac{\theta\left(\zeta^{\prime}-z-z_{i} z^{\prime}\right)}{\left[\kappa^{2}\left(1-z^{2}\right)+m^{2} z^{2}+\gamma-i \epsilon\right]} \\
& \quad \times \frac{1}{\kappa^{2}\left(1-z^{2}\right)+m^{2} z^{2}+\gamma+\frac{(1+z)\left(\frac{M^{2}}{2} z z^{\prime} z_{i}+\gamma^{\prime}\right)}{\left(1+\zeta^{\prime}-z^{\prime} z_{i}\right)}-i \epsilon} \\
& =\theta\left(\zeta^{\prime}-z-z_{i} z^{\prime}\right) \int_{0}^{1} \mathrm{~d} \xi \\
& \quad \times \frac{1}{\left[\gamma+m^{2} z^{2}+\kappa^{2}\left(1-z^{2}\right)+\xi \frac{(1+z)\left(\frac{M^{2}}{2} z z^{\prime} z_{i}+\gamma^{\prime}\right)}{\left(1+\zeta^{\prime}-z^{\prime} z_{i}\right)}-i \epsilon\right]^{2}} \\
& =\frac{\left(1+\zeta^{\prime}-z^{\prime} z_{i}\right)}{(1+z)} \int_{0}^{1} \mathrm{~d} \alpha \theta\left[\frac{(1+z)}{\left(1+\zeta^{\prime}-z^{\prime} z_{i}\right)}-\alpha\right] \\
& \quad \times \frac{\theta\left(\zeta^{\prime}-z-z_{i} z^{\prime}\right)}{\left[\gamma+m^{2} z^{2}+\kappa^{2}\left(1-z^{2}\right)+\alpha\left(\frac{M^{2}}{2} z z^{\prime} z_{i}+\gamma^{\prime}\right)-i \epsilon\right]^{2}},
\end{aligned}
$$

with

$1 \geq \frac{(1+z)}{\left(1+\zeta^{\prime}-z^{\prime} z_{i}\right)}=\frac{(1+z)}{\left[1+z+\left(\zeta^{\prime}-z-z^{\prime} z_{i}\right)\right]}$,

since $\theta\left(\zeta^{\prime}-z-z_{i} z^{\prime}\right)$. Inserting the above expression, together with the one containing $\theta\left(z+z_{i} z^{\prime}-\zeta^{\prime}\right)$, in Eq. (B.15), one gets the following expression for the distorted term:

$$
\begin{aligned}
\psi_{\mathrm{dist}}\left(z, \gamma ; z_{i}, \kappa^{2}\right) & i \frac{\left(1-z^{2}\right)}{4} \int_{-1}^{1} \mathrm{~d} \zeta^{\prime \prime} \int_{-1}^{1} \mathrm{~d} \zeta^{\prime} \int_{-\infty}^{\infty} \mathrm{d} \gamma^{\prime \prime} \int_{0}^{1} \frac{\mathrm{d} \alpha}{\alpha^{2}} \\
= & \times \theta\left(\alpha-\left|\zeta^{\prime \prime}\right|\right) \theta\left(1-\left|\frac{\zeta^{\prime \prime}}{\alpha}\right|-\left|\zeta^{\prime}\right|\right) \\
& \times \frac{\widetilde{\mathcal{G}}^{+}\left(\frac{\gamma^{\prime \prime}}{\alpha}, \frac{\zeta^{\prime \prime}}{\alpha}, \zeta^{\prime}\right)}{\left[\gamma+m^{2} z^{2}+\kappa^{2}\left(1-z^{2}\right)+\gamma^{\prime \prime}+\frac{M^{2}}{2} z \zeta^{\prime \prime} z_{i}-i \epsilon\right]^{2}} \\
& \times\left\{\theta\left[\left(1+z+\zeta^{\prime \prime} z_{i}\right)-\alpha\left(1+\zeta^{\prime}\right)\right] \theta\left[\alpha\left(\zeta^{\prime}-z\right)-z_{i} \zeta^{\prime \prime}\right]\right. \\
& \left.\left.+\theta\left[\left(1-z-\zeta^{\prime \prime} z_{i}\right)-\alpha\left(1-\zeta^{\prime}\right)\right)\right] \theta\left[-\alpha\left(\zeta^{\prime}-z\right)+z_{i} \zeta^{\prime \prime}\right]\right\} \\
= & i \frac{\left(1-z^{2}\right)}{4} \int_{-1}^{1} \mathrm{~d} \zeta^{\prime \prime} \int_{-\infty}^{\infty} \mathrm{d} \gamma^{\prime \prime} \int_{0}^{1} \frac{\mathrm{d} \alpha}{\alpha^{3}} \int_{-1}^{1} \mathrm{~d} y \\
& \times \theta(\alpha-|y|) \theta\left(\alpha-\left|\zeta^{\prime \prime}\right|\right) \theta\left(\alpha-\left|\zeta^{\prime \prime}\right|-|y|\right) \\
& \times \frac{\widetilde{\mathcal{G}}^{+}\left(\frac{\gamma^{\prime \prime}}{\alpha}, \frac{\zeta^{\prime \prime}}{\alpha}, \frac{y}{\alpha}\right)}{\left[\gamma+m^{2} z^{2}+\kappa^{2}\left(1-z^{2}\right)+\gamma^{\prime \prime}+\frac{M^{2}}{2} z \zeta^{\prime \prime} z_{i}-i \epsilon\right]^{2}}
\end{aligned}
$$




$$
\begin{aligned}
& \times\left\{\theta\left(1+z+\zeta^{\prime \prime} z_{i}-\alpha-y\right) \theta\left(y-\alpha z-z_{i} \zeta^{\prime \prime}\right)+\right. \\
& \left.\theta\left(1-z-\zeta^{\prime \prime} z_{i}-\alpha+y\right] \theta\left[-y+\alpha z+z_{i} \zeta^{\prime \prime}\right)\right\}
\end{aligned}
$$

where $\gamma^{\prime \prime}=\alpha \gamma^{\prime}$ and $\zeta^{\prime \prime}=\alpha z^{\prime}$.

The theta functions between curly brackets single out the following integration regions:

$-1-\alpha+z+\zeta^{\prime \prime} z_{i} \geq y \geq \alpha z+z_{i} \zeta^{\prime \prime}$,

$-\alpha z+z_{i} \zeta^{\prime \prime} \geq y \geq-(1-\alpha)+z+\zeta^{\prime \prime} z_{i}$.

The above intervals lead to the following constraint:

$1-\alpha \geq y-z-\zeta^{\prime \prime} z_{i} \geq-(1-\alpha)$

namely $\theta\left(1-\alpha-\left|y-z-\zeta^{\prime \prime} z_{i}\right|\right)$. Then one gets

$$
\begin{aligned}
& \psi_{\text {dist }}\left(z, \gamma ; z_{i}, \kappa^{2}\right)=i \frac{\left(1-z^{2}\right)}{4} \int_{-1}^{1} \mathrm{~d} \zeta^{\prime \prime} \int_{-\infty}^{\infty} \mathrm{d} \gamma^{\prime \prime} \\
& \times \int_{0}^{1} \frac{\mathrm{d} \alpha}{\alpha^{3}} \int_{-1}^{1} \mathrm{~d} y \widetilde{\mathcal{G}}^{+}\left(\frac{\gamma^{\prime \prime}}{\alpha}, \frac{\zeta^{\prime \prime}}{\alpha}, \frac{y}{\alpha}\right) \\
& \times \frac{\theta\left(\alpha-\left|\zeta^{\prime \prime}\right|-|y|\right) \theta\left(1-\alpha-\left|y-z-\zeta^{\prime \prime} z_{i}\right|\right)}{\left[\gamma+m^{2} z^{2}+\kappa^{2}\left(1-z^{2}\right)+\gamma^{\prime \prime}+\frac{M^{2}}{2} z \zeta^{\prime \prime} z_{i}-i \epsilon\right]^{2}} .
\end{aligned}
$$

The above expression of $\psi_{\text {dist }}$ allows one to write the following relation between the Nakanishi weight function $g^{+}\left(\gamma^{\prime}, z^{\prime}, z ; \kappa^{2}, z_{i}\right)$, which appears in Eq. (B.6), and $\widetilde{\mathcal{G}}^{+}$, namely the Nakanishi weight function involved in the description the half-off-shell T-matrix:

$$
\begin{aligned}
g^{+} & \left(\gamma^{\prime}, z^{\prime}, z ; \kappa^{2}, z_{i}\right) \\
= & i \int_{0}^{1} \frac{\mathrm{d} \alpha}{\alpha^{3}} \int_{-1}^{1} \mathrm{~d} y \widetilde{\mathcal{G}}^{+}\left(\frac{\gamma^{\prime}}{\alpha}, \frac{z^{\prime}}{\alpha}, \frac{y}{\alpha} ; \kappa^{2}, z_{i}\right) \\
& \times \theta\left(\alpha-\left|z^{\prime}\right|-|y|\right) \theta\left(1-\alpha-\left|y-z-z^{\prime} z_{i}\right|\right) .
\end{aligned}
$$

Notice that Eq. (B.19) can be transformed into Eq. (A.4) by applying a suitable change of variables.

\section{Appendix C: The support of the Nakanishi weight func- tion for scattering states}

This appendix is devoted to a thorough discussion of the support issue for the Nakanishi weight function, in the case of the ladder inhomogeneous Bethe-Salpeter equation.

Let us first rewrite the integral equation for the Nakanishi weight, obtained after invoking the uniqueness theorem [12]. It reads

$$
\begin{aligned}
& g_{(L d)}^{(+)}\left(\gamma, z^{\prime}, z ; \kappa^{2}, z_{i}\right)=g^{2} \theta\left(-z^{\prime}\right) \delta\left(\gamma-\gamma_{a}\left(z^{\prime}\right)\right) \\
& \quad \times\left\{\theta\left(z-z_{i}\right) \theta\left[1-z+z^{\prime}\left(1-z_{i}\right)\right]\right. \\
& \left.\quad+\theta\left(z_{i}-z\right) \theta\left[1+z+z^{\prime}\left(1+z_{i}\right)\right]\right\}+
\end{aligned}
$$

$$
\begin{aligned}
& -\frac{g^{2}}{2(4 \pi)^{2}} \int_{-\infty}^{\infty} \mathrm{d} \gamma^{\prime} \int_{-1}^{1} \mathrm{~d} \zeta \int_{-1}^{1} \mathrm{~d} \zeta^{\prime} g_{(L d)}^{(+)}\left(\gamma^{\prime}, \zeta, \zeta^{\prime} ; \kappa^{2}, z_{i}\right) \\
& \times\left[\frac{(1+z) \theta\left(\zeta^{\prime}-z-z_{i} \zeta\right)}{\left(1+\zeta^{\prime}-z_{i} \zeta\right)} h^{\prime}\left(\gamma, z^{\prime}, z, z_{i} ; \gamma^{\prime}, \zeta, \zeta^{\prime}, \mu^{2}\right)\right. \\
& \left.+\frac{(1-z) \theta\left(z-\zeta^{\prime}+z_{i} \zeta\right)}{\left(1-\zeta^{\prime}+z_{i} \zeta\right)} h^{\prime}\left(\gamma, z^{\prime},-z,-z_{i} ; \gamma^{\prime}, \zeta,-\zeta^{\prime}, \mu^{2}\right)\right]
\end{aligned}
$$

where

$\gamma_{a}\left(z^{\prime}\right)=z^{\prime}\left(2 \kappa^{2}-\mu^{2}\right) \geq 0$

and $h^{\prime}\left(\gamma^{\prime \prime}, z^{\prime}, z, z_{i} ; \gamma^{\prime}, \zeta, \zeta^{\prime}, \mu^{2}\right)$ is given by

$$
\begin{aligned}
& h^{\prime}\left(\gamma^{\prime \prime}, z^{\prime}, z, z_{i} ; \gamma^{\prime}, \zeta, \zeta^{\prime}, \mu^{2}\right)=\frac{(1+z)}{\left(1+\zeta^{\prime}-z_{i} \zeta\right)} \\
& \quad \times\left\{\frac{\partial}{\partial \lambda} \int_{0}^{\infty} \mathrm{d} y \int_{0}^{1} \mathrm{~d} \xi \delta\left[z^{\prime}-\xi Z\left(z, \zeta, \zeta^{\prime} ; z_{i}\right)\right]\right. \\
& \left.\quad \times \delta\left[\mathcal{F}\left(\lambda, y, \xi ; \gamma^{\prime \prime}, z, \zeta, \zeta^{\prime}, \gamma^{\prime} ; z_{i}, \kappa^{2}, \mu^{2}\right)\right]\right\}_{\lambda=0},
\end{aligned}
$$

with

$$
\begin{aligned}
\mathcal{F} & \left(\lambda, y, \xi ; \gamma^{\prime \prime}, z, \zeta, \zeta^{\prime}, \gamma^{\prime} ; z_{i}, \kappa^{2}, \mu^{2}\right) \\
= & \gamma^{\prime \prime}-\xi \frac{(1+z)}{\left(1+\zeta^{\prime}-z_{i} \zeta\right)} \\
& \times\left(\frac{y^{2} \mathcal{A}\left(\zeta, \zeta^{\prime}, \gamma^{\prime}, \kappa^{2}\right)+y\left(\mu^{2}+\gamma^{\prime}\right)+\mu^{2}}{y}\right)-\xi \lambda
\end{aligned}
$$

For $\kappa^{2}<0$ the support in $\gamma$ is $(-\infty, \infty)$, and in order to carefully analyze the behavior of $g_{(L d)}^{(+)}\left(\gamma, z^{\prime}, z ; \kappa^{2}, z_{i}\right)$, it is useful to split the integral equation (C.20) in two coupled integral equations: one as a result is found to be inhomogeneous, while the other turns out to be a homogeneous one. To show this, let us introduce the following decomposition of the weight function $g_{(L d)}^{(+)}\left(\gamma, z^{\prime}, z ; \kappa^{2}, z_{i}\right)$ :

$$
\begin{aligned}
& g_{(L d)}^{(+)}\left(\gamma, z^{\prime}, z ; \kappa^{2}, z_{i}\right)=\theta(\gamma) g_{p ;(L d)}^{(+)}\left(\gamma, z^{\prime}, z ; \kappa^{2}, z_{i}\right) \\
& \quad+\theta(-\gamma) g_{n ;(L d)}^{(+)}\left(\gamma, z^{\prime}, z ; \kappa^{2}, z_{i}\right) .
\end{aligned}
$$

Inserting such a decomposition in Eq. (C.20) one gets

$$
\begin{aligned}
& g_{p ;(L d)}^{(+)}\left(\gamma, z^{\prime}, z ; \kappa^{2}, z_{i}\right)=g^{2} \theta\left(-z^{\prime}\right) \delta\left(\gamma-\gamma_{a}\left(z^{\prime}\right)\right) \\
& \quad \times\left\{\theta\left(z-z_{i}\right) \theta\left[1-z+z^{\prime}\left(1-z_{i}\right)\right]\right. \\
& \left.\quad+\theta\left(z_{i}-z\right) \theta\left[1+z+z^{\prime}\left(1+z_{i}\right)\right]\right\}+ \\
& \quad-\frac{g^{2}}{2(4 \pi)^{2}} \theta(\gamma)\left[\int_{0}^{\infty} \mathrm{d} \gamma^{\prime} \int_{-1}^{1} \mathrm{~d} \zeta \int_{-1}^{1} \mathrm{~d} \zeta^{\prime}\right. \\
& \quad \times H^{\prime}\left(\gamma, z^{\prime}, z, z_{i} ; \gamma^{\prime}, \zeta, \zeta^{\prime}, \mu^{2}\right) g_{p ;(L d)}^{(+)}\left(\gamma^{\prime}, \zeta, \zeta^{\prime} ; \kappa^{2}, z_{i}\right)
\end{aligned}
$$




$$
\begin{aligned}
& +\int_{-\infty}^{0} \mathrm{~d} \gamma^{\prime} \int_{-1}^{1} \mathrm{~d} \zeta \int_{-1}^{1} \mathrm{~d} \zeta^{\prime} \\
& \left.\times H^{\prime}\left(\gamma, z^{\prime}, z, z_{i} ; \gamma^{\prime}, \zeta, \zeta^{\prime}, \mu^{2}\right) g_{n ;(L d)}^{(+)}\left(\gamma^{\prime}, \zeta, \zeta^{\prime} ; \kappa^{2}, z_{i}\right)\right]
\end{aligned}
$$

and

$$
\begin{aligned}
& g_{n ;(L d)}^{(+)}\left(\gamma, z^{\prime}, z ; \kappa^{2}, z_{i}\right)=-\frac{g^{2}}{2(4 \pi)^{2}} \theta(-\gamma) \\
& \quad \times\left[\int_{0}^{\infty} \mathrm{d} \gamma^{\prime} \int_{-1}^{1} \mathrm{~d} \zeta \int_{-1}^{1} \mathrm{~d} \zeta^{\prime}\right. \\
& \quad \times H^{\prime}\left(\gamma, z^{\prime}, z, z_{i} ; \gamma^{\prime}, \zeta, \zeta^{\prime}, \mu^{2}\right) g_{p ;(L d)}^{(+)}\left(\gamma^{\prime}, \zeta, \zeta^{\prime} ; \kappa^{2}, z_{i}\right) \\
& \quad+\int_{-\infty}^{0} \mathrm{~d} \gamma^{\prime} \int_{-1}^{1} \mathrm{~d} \zeta \int_{-1}^{1} \mathrm{~d} \zeta^{\prime} \\
& \left.\quad \times H^{\prime}\left(\gamma, z^{\prime}, z, z_{i} ; \gamma^{\prime}, \zeta, \zeta^{\prime}, \mu^{2}\right) g_{n ;(L d)}^{(+)}\left(\gamma^{\prime}, \zeta, \zeta^{\prime} ; \kappa^{2}, z_{i}\right)\right],
\end{aligned}
$$

with

$$
\begin{aligned}
& H^{\prime}\left(\gamma, z^{\prime}, z, z_{i} ; \gamma^{\prime}, \zeta, \zeta^{\prime}, \mu^{2}\right)= \\
& =\left[\frac{(1+z) \theta\left(\zeta^{\prime}-z-z_{i} \zeta\right)}{\left(1+\zeta^{\prime}-z_{i} \zeta\right)} h^{\prime}\left(\gamma, z^{\prime}, z, z_{i} ; \gamma^{\prime}, \zeta, \zeta^{\prime}, \mu^{2}\right)\right. \\
& \quad+\frac{(1-z) \theta\left(z-\zeta^{\prime}+z_{i} \zeta\right)}{\left(1-\zeta^{\prime}+z_{i} \zeta\right)} \\
& \left.\quad \times h^{\prime}\left(\gamma, z^{\prime},-z,-z_{i} ; \gamma^{\prime}, \zeta,-\zeta^{\prime}, \mu^{2}\right)\right] .
\end{aligned}
$$

If $\kappa^{2} \rightarrow 0^{-}$, the off-shell kernel in the homogeneous integral equation, namely the one with $\gamma<0$ and $\gamma^{\prime}>0$, becomes vanishing and this leads to a system of uncoupled equations. As a matter of fact, one has for $\kappa^{2} \rightarrow 0^{-}$

$$
\begin{aligned}
& \theta(-\gamma) \theta\left(\gamma^{\prime}\right) H^{\prime}\left(\gamma, z^{\prime}, z, z_{i}=0 ; \gamma^{\prime}, \zeta, \zeta^{\prime}, \mu^{2}\right) \\
&= \theta(-\gamma) \theta\left(\gamma^{\prime}\right) \frac{(1+z)}{\left(1+\zeta^{\prime}\right)}\left\{\frac{\partial}{\partial \lambda} \int_{0}^{\infty} \mathrm{d} y\right. \\
& \quad \times \int_{0}^{1} \mathrm{~d} \xi \delta\left[z^{\prime}-\xi \zeta \frac{(1+z)}{\left(1+\zeta^{\prime}\right)}\right] \\
&\left.\times \delta\left[\mathcal{F}\left(\lambda, y, \xi ; \gamma^{\prime \prime}, z, \zeta, \zeta^{\prime}, \gamma^{\prime} ; z_{i}, \kappa^{2}=0, \mu^{2}\right)\right]\right\}_{\lambda=0} \\
&= 0
\end{aligned}
$$

since the delta function is always vanishing, given $\gamma<0$ and

$\xi \frac{(1+z)}{\left(1+\zeta^{\prime}\right)}\left(\frac{y^{2} \mathcal{A}\left(\zeta, \zeta^{\prime}, \gamma^{\prime}, \kappa^{2}=0\right)+y\left(\mu^{2}+\gamma^{\prime}\right)+\mu^{2}}{y}\right)$

$+\xi \lambda$

$=\xi \frac{(1+z)}{\left(1+\zeta^{\prime}\right)}\left[\frac{y^{2}\left(\zeta^{\prime 2} m^{2}+\gamma^{\prime}\right)+y\left(\mu^{2}+\gamma^{\prime}\right)+\mu^{2}}{y}\right]$

$+\xi \lambda>0$.
Then, for $\kappa^{2} \rightarrow 0^{-}$, Eq. (C.26) becomes

$$
\begin{aligned}
& g_{n ;(L d)}^{(+)}\left(\gamma, z^{\prime}, z ; \kappa^{2}=z_{i}=0\right)=-\frac{g^{2}}{2(4 \pi)^{2}} \theta(-\gamma) \\
& \quad \int_{-\infty}^{0} \mathrm{~d} \gamma^{\prime} \int_{-1}^{1} \mathrm{~d} \zeta \int_{-1}^{1} \mathrm{~d} \zeta^{\prime} H^{\prime}\left(\gamma, z^{\prime}, z, z_{i} ; \gamma^{\prime}, \zeta, \zeta^{\prime}, \mu^{2}\right) \\
& \quad \times g_{n ;(L d)}^{(+)}\left(\gamma^{\prime}, \zeta, \zeta^{\prime} ; \kappa^{2}=z_{i}=0\right) .
\end{aligned}
$$

The above homogeneous integral equation (we recall that it holds in the zero-energy limit) has a vanishing solution, $g_{n ;(L d)}^{(+)}\left(\gamma, z^{\prime}, z ; \kappa^{2}=z_{i}=0\right)=0$, given the freedom in choosing $g^{2}$ for scattering states. Therefore one is left with only the component $g_{p ;(L d)}^{(+)}\left(\gamma, z^{\prime}, z ; \kappa^{2}=z_{i}=0\right)$.

It is also instructive to trace the behavior of the previous coupling term when $\kappa^{2}$ approaches $0^{-}$. If $\kappa^{2}$ is different from zero, than the delta function in Eq. (C.28) can give a finite contribution, since its argument can vanish. To achieve such a possibility, one must have (recall that $\gamma^{\prime}>0$ )

$\mathcal{A}\left(\zeta, \zeta^{\prime}, \gamma^{\prime}, \kappa^{2}\right)=\frac{M^{2}}{4} \zeta^{\prime 2}+\kappa^{2}\left(1+\zeta^{2}\right)+\gamma^{\prime}<0$,

since the other terms, $\mu^{2}+\gamma^{\prime}$ and $\lambda$, always yield a positive contribution ( $\lambda$ approaches zero from positive values). The above constraint leads to a volume of the integration in the space $\left\{\gamma^{\prime}, \zeta^{\prime}, \zeta\right\}$ (it is a hyperboloid), which shrinks to zero for $\kappa^{2} \rightarrow 0^{-}$, viz.

$\frac{M^{2}}{4} \zeta^{\prime 2}+\kappa^{2} \zeta^{2}+\gamma^{\prime}<-\kappa^{2}$.

\section{Appendix D: Zero-energy limit}

The zero-energy limit of the relevant integral equations fulfilled by the Nakanishi weight function amounts to consider the case $\kappa^{2}=0$, namely $M^{2}=4 m^{2}$. This entails $\gamma_{i}=z_{i}=0$ through $M^{2}=4\left(m^{2}+\gamma_{i}\right) /\left(1-z_{i}^{2}\right)$. In this appendix, the integral equations obtained both without applying the uniqueness theorem [12] and by exploiting it, are obtained following a simpler procedure than the one adopted in Ref. [9] (notice that a mistyping present in Eq. (103) of [9] has been fixed in this appendix, as explained in what follows).

The Nakanishi integral equation, involving $\psi_{\text {dist }}$, for $\kappa^{2} \leq$ 0 (see [9]) is given by

$$
\begin{aligned}
& \int_{-1}^{1} \mathrm{~d} z^{\prime} \int_{-\infty}^{\infty} \mathrm{d} \gamma^{\prime \prime} g_{(L d)}^{(+)}\left(\gamma^{\prime \prime}, z^{\prime}, z ; \gamma_{i}, z_{i}\right) \\
& \quad \times \frac{1}{\left[\gamma+\gamma^{\prime \prime}+z^{2} m^{2}+\left(1-z^{2}\right) \kappa^{2}+z^{\prime} \frac{M^{2}}{2} z z_{i}-i \epsilon\right]^{2}} \\
& =g^{2} \int_{-1}^{1} \mathrm{~d} z^{\prime} \int_{-\infty}^{\infty} \mathrm{d} \gamma^{\prime \prime} \theta\left(-z^{\prime}\right) \delta\left(\gamma^{\prime \prime}-\gamma_{a}\left(z^{\prime}\right)\right) \\
& \quad \times \frac{1}{\left[\gamma+\gamma^{\prime \prime}+\left(1-z^{2}\right) \kappa^{2}+z^{2} m^{2}+z^{\prime} \frac{M^{2}}{2} z z_{i}-i \epsilon\right]^{2}}
\end{aligned}
$$




$$
\begin{aligned}
& \left\{\theta\left(z-z_{i}\right) \theta\left[1-z+z^{\prime}\left(1-z_{i}\right)\right]\right. \\
& \left.+\theta\left(z_{i}-z\right) \theta\left[1+z+z^{\prime}\left(1+z_{i}\right)\right]\right\} \\
& -\frac{g^{2}}{2(4 \pi)^{2}} \int_{-\infty}^{\infty} \mathrm{d} \gamma^{\prime \prime} \int_{-1}^{1} \mathrm{~d} z^{\prime} \\
& \times \frac{1}{\left[\gamma+\gamma^{\prime \prime}+z^{2} m^{2}+\kappa^{2}\left(1-z^{2}\right)+z^{\prime} \frac{M^{2}}{2} z z_{i}-i \epsilon\right]^{2}} \\
& \int_{-\infty}^{\infty} \mathrm{d} \gamma^{\prime} \int_{-1}^{1} \mathrm{~d} \zeta \int_{-1}^{1} \mathrm{~d} \zeta^{\prime} g_{(L d)}^{(+)}\left(\gamma^{\prime}, \zeta, \zeta^{\prime} ; \kappa^{2}, z_{i}\right) \\
& \times\left[\frac{(1+z)}{\left(1+\zeta^{\prime}-z_{i} \zeta\right)}\right. \\
& \times \theta\left(\zeta^{\prime}-z-z_{i} \zeta\right) h^{\prime}\left(\gamma^{\prime \prime}, z^{\prime}, z, z_{i} ; \gamma^{\prime}, \zeta, \zeta^{\prime}, \mu^{2}\right) \\
& +\frac{(1-z)}{\left(1-\zeta^{\prime}+z_{i} \zeta\right)} \\
& \left.\times \theta\left(z-\zeta^{\prime}+z_{i} \zeta\right) h^{\prime}\left(\gamma^{\prime \prime}, z^{\prime},-z,-z_{i} ; \gamma^{\prime}, \zeta,-\zeta^{\prime}, \mu^{2}\right)\right],
\end{aligned}
$$

with

$$
\begin{aligned}
& h^{\prime}\left(\gamma^{\prime \prime}, z^{\prime}, z, z_{i} ; \gamma^{\prime}, \zeta, \zeta^{\prime}, \mu^{2}\right)=\frac{(1+z)}{\left(1+\zeta^{\prime}-z_{i} \zeta\right)} \\
& \quad \times\left\{\frac{\partial}{\partial \lambda} \int_{0}^{\infty} \mathrm{d} y \int_{0}^{1} \mathrm{~d} \xi \delta\left[z^{\prime}-\xi Z\left(z, \zeta, \zeta^{\prime} ; z_{i}\right)\right]\right. \\
& \left.\quad \times \delta\left[\mathcal{F}\left(\lambda, y, \xi ; \gamma^{\prime \prime}, z, \zeta, \zeta^{\prime}, \gamma^{\prime} ; z_{i}, \kappa^{2}, \mu^{2}\right)\right]\right\}_{\lambda=0},
\end{aligned}
$$

where

$$
\begin{aligned}
\mathcal{F} & \left(\lambda, y, \xi ; \gamma^{\prime \prime}, z, \zeta, \zeta^{\prime}, \gamma^{\prime} ; z_{i}, \kappa^{2}, \mu^{2}\right) \\
= & \gamma^{\prime \prime}-\xi \frac{(1+z)}{\left(1+\zeta^{\prime}-z_{i} \zeta\right)} \\
& \times\left(\frac{y^{2} \mathcal{A}\left(\zeta, \zeta^{\prime}, \gamma^{\prime}, \kappa^{2}\right)+y\left(\mu^{2}+\gamma^{\prime}\right)+\mu^{2}}{y}\right)-\xi \lambda .
\end{aligned}
$$

For $\kappa^{2}=0$, it follows that $\gamma_{i}=z_{i}=0$, since

$\kappa^{2}=m^{2}-\frac{M^{2}}{4}=\left(\frac{p}{2} \pm k_{i}\right)^{2}-\frac{M^{2}}{4}=-\gamma_{i}-z_{i}^{2} \frac{M^{2}}{4}=0$.

Then, taking into account that $g_{(L d)}^{(+)}\left(\gamma, z^{\prime}, z ; \kappa^{2}=0\right)$ has support only for positive $\gamma$, one can write [cf. Eq. (D.31) and Sect. 2.2]

$$
\begin{aligned}
& \int_{-1}^{1} \mathrm{~d} z^{\prime} \int_{0}^{\infty} \mathrm{d} \gamma^{\prime \prime} \frac{g_{(L d)}^{(+)}\left(\gamma^{\prime \prime}, z^{\prime}, z ; \kappa^{2}=0\right)}{\left[\gamma+\gamma^{\prime \prime}+z^{2} m^{2}-i \epsilon\right]^{2}}= \\
& =g^{2} \int_{0}^{\infty} \mathrm{d} \gamma^{\prime \prime} \int_{-1}^{1} \mathrm{~d} z^{\prime} \frac{\theta\left(-z^{\prime}\right) \delta\left(\gamma^{\prime \prime}+z^{\prime} \mu^{2}\right)}{\left[\gamma+\gamma^{\prime \prime}+z^{2} m^{2}-i \epsilon\right]^{2}} \\
& \quad \times\left[\theta(z) \theta\left(1-z+z^{\prime}\right)+\theta(-z) \theta\left(1+z+z^{\prime}\right)\right]
\end{aligned}
$$

$$
\begin{aligned}
& -\frac{g^{2}}{2(4 \pi)^{2}} \int_{0}^{\infty} \mathrm{d} \gamma^{\prime} \int_{-1}^{1} \mathrm{~d} \zeta \\
& \times \int_{-1}^{1} \mathrm{~d} \zeta^{\prime} g_{(L d)}^{(+)}\left(\gamma^{\prime}, \zeta, \zeta^{\prime} ; \kappa^{2}=0\right) \\
& \times \int_{-\infty}^{\infty} \mathrm{d} \gamma^{\prime \prime} \frac{1}{\left[\gamma+\gamma^{\prime \prime}+z^{2} m^{2}-i \epsilon\right]^{2}} \int_{-1}^{1} \mathrm{~d} z^{\prime} \\
& \times\left[\frac{(1+z)}{\left(1+\zeta^{\prime}\right)} \theta\left(\zeta^{\prime}-z\right) \mathcal{Z}^{\prime}\left(\gamma^{\prime \prime}, z^{\prime}, z ; \gamma^{\prime}, \zeta^{\prime}, \mu^{2}\right)\right. \\
& \left.+\frac{(1-z)}{\left(1-\zeta^{\prime}\right)} \theta\left(z-\zeta^{\prime}\right) \mathcal{Z}^{\prime}\left(\gamma^{\prime \prime}, z^{\prime},-z ; \gamma^{\prime},-\zeta^{\prime}, \mu^{2}\right)\right]
\end{aligned}
$$

where the kernel $\mathcal{Z}^{\prime}$ is given by

$$
\begin{aligned}
& \mathcal{Z}^{\prime}\left(\gamma^{\prime \prime}, z^{\prime}, z ; \gamma^{\prime}, \zeta^{\prime}, \mu^{2}\right)=\frac{(1+z)}{\left(1+\zeta^{\prime}\right)} \\
& \quad \times\left\{\frac{\partial}{\partial \lambda} \int_{0}^{\infty} d y \int_{0}^{1} \mathrm{~d} \xi \delta\left[\gamma^{\prime \prime}-\xi \Gamma_{0}\left(y, z, \zeta^{\prime}, \gamma^{\prime}\right)-\xi \lambda\right]\right. \\
& \left.\quad \times \delta\left[z^{\prime}-\xi \frac{(1+z)}{\left(1+\zeta^{\prime}\right)} \zeta\right]\right\}_{\lambda=0}
\end{aligned}
$$

with

$$
\begin{aligned}
& \Gamma_{0}\left(y, z, \zeta^{\prime}, \gamma^{\prime}\right)=\frac{(1+z)}{\left(1+\zeta^{\prime}\right)} \frac{1}{y} \\
& \quad \times\left\{y^{2} \mathcal{A}_{0}\left(\zeta^{\prime}, \gamma^{\prime}\right)+y\left(\mu^{2}+\gamma^{\prime}\right)+\mu^{2}\right\}
\end{aligned}
$$

and

$\mathcal{A}_{0}\left(\zeta^{\prime}, \gamma^{\prime}\right)=\zeta^{\prime 2} \frac{M^{2}}{4}+\gamma^{\prime}=\zeta^{\prime 2} m^{2}+\gamma^{\prime} \geq 0$.

Notice that $\gamma^{\prime}$ is positive and therefore also $\Gamma_{0}$ has to be positive. Finally, $\gamma^{\prime \prime}$ in Eq. (D.35) has to be positive for getting a non-vanishing $\mathcal{Z}^{\prime}\left(\gamma^{\prime \prime}, z^{\prime}, z ; \gamma^{\prime}, \zeta^{\prime}, \mu^{2}\right)$.

Performing (i) the integration on $z^{\prime}$ in both sides of Eq. (D.34) (recall that $\left.1>\left|\xi \zeta(1 \pm z) /\left(1 \pm \zeta^{\prime}\right)\right|\right)$, and (ii) the integration on $\zeta$ in the rhs, one gets

$$
\begin{aligned}
& \int_{0}^{\infty} \mathrm{d} \gamma^{\prime \prime} \frac{g_{0 L d}^{(+)}\left(\gamma^{\prime \prime}, z\right)}{\left[\gamma+\gamma^{\prime \prime}+z^{2} m^{2}-i \epsilon\right]^{2}}= \\
& =\frac{g^{2}}{\mu^{2}} \int_{-\infty}^{\infty} \mathrm{d} \gamma^{\prime \prime} \frac{\theta\left(\gamma^{\prime \prime}\right)}{\left[\gamma+\gamma^{\prime \prime}+z^{2} m^{2}-i \epsilon\right]^{2}} \\
& \quad \times\left\{\theta(z) \theta\left[1-z-\gamma^{\prime \prime} / \mu^{2}\right]\right. \\
& \left.\quad+\theta(-z) \theta\left[1+z-\gamma^{\prime \prime} / \mu^{2}\right]\right\} \\
& \quad-\frac{g^{2}}{2(4 \pi)^{2}} \int_{0}^{\infty} \mathrm{d} \gamma^{\prime} \int_{-1}^{1} \mathrm{~d} \zeta^{\prime} g_{0 L d}^{(+)}\left(\gamma^{\prime}, \zeta^{\prime}\right) \\
& \quad \times \int_{-\infty}^{\infty} \mathrm{d} \gamma^{\prime \prime} \theta\left(\gamma^{\prime \prime}\right) \frac{1}{\left[\gamma+\gamma^{\prime \prime}+z^{2} m^{2}-i \epsilon\right]^{2}}
\end{aligned}
$$




$$
\begin{aligned}
& \times\left[\frac{(1+z)}{\left(1+\zeta^{\prime}\right)} \theta\left(\zeta^{\prime}-z\right) h_{0}^{\prime}\left(\gamma^{\prime \prime}, z ; \gamma^{\prime}, \zeta^{\prime}, \mu^{2}\right)\right. \\
& \left.+\frac{(1-z)}{\left(1-\zeta^{\prime}\right)} \theta\left(z-\zeta^{\prime}\right) h_{0}^{\prime}\left(\gamma^{\prime \prime},-z ; \gamma^{\prime},-\zeta^{\prime}, \mu^{2}\right)\right]
\end{aligned}
$$

where:

1.

$$
g_{0 L d}^{(+)}\left(\gamma^{\prime \prime}, z\right)=\int_{-1}^{1} \mathrm{~d} z^{\prime} g_{(L d)}^{(+)}\left(\gamma^{\prime \prime}, z^{\prime}, z ; \kappa^{2}=0\right) .
$$

2.

$$
\int_{-1}^{1} \mathrm{~d} z^{\prime} \theta\left(-z^{\prime}\right) \delta\left(\gamma^{\prime \prime}+z^{\prime} \mu^{2}\right)=\frac{1}{\mu^{2}} \theta\left(\gamma^{\prime \prime}\right) \theta\left(\mu^{2}-\gamma^{\prime \prime}\right) .
$$

3.

$$
\begin{gathered}
h_{0}^{\prime}\left(\gamma^{\prime \prime}, z ; \gamma^{\prime}, \zeta^{\prime}, \mu^{2}\right)=\frac{(1+z)}{\left(1+\zeta^{\prime}\right)} \frac{\partial}{\partial \lambda} \int_{0}^{\infty} d y \\
\left.\quad \int_{0}^{1} \mathrm{~d} \xi \delta\left[\gamma^{\prime \prime}-\xi \Gamma_{0}\left(y, z, \zeta^{\prime}, \gamma^{\prime}\right)-\xi \lambda\right]\right|_{\lambda=0} .
\end{gathered}
$$

From Ref. [10], one recognizes that $h_{0}^{\prime}\left(\gamma^{\prime \prime}, z ; \gamma^{\prime}, \zeta^{\prime}, \mu^{2}\right)$ is the suitable kernel for a bound state with zero energy. Therefore one can write

$$
\begin{aligned}
h_{0}^{\prime}\left(\gamma^{\prime \prime}, z ; \gamma^{\prime}, \zeta^{\prime}, \mu^{2}\right) \\
=\theta\left[-\mathcal{B}_{0}\left(z, \zeta^{\prime}, \gamma^{\prime}, \gamma^{\prime \prime}, \mu^{2}\right)-2 \mu \sqrt{\zeta^{\prime 2} \frac{M^{2}}{4}+\gamma^{\prime}}\right] \\
\quad \times\left[-\frac{\mathcal{B}_{0}\left(z, \zeta^{\prime}, \gamma^{\prime}, \gamma^{\prime \prime}, \mu^{2}\right)}{\mathcal{A}_{0}\left(\zeta^{\prime}, \gamma^{\prime}\right) \Delta_{0}\left(z, \zeta^{\prime}, \gamma^{\prime}, \gamma^{\prime \prime}, \mu^{2}\right)} \frac{1}{\gamma^{\prime \prime}}+\frac{\left(1+\zeta^{\prime}\right)}{(1+z)}\right. \\
\left.\quad \times \int_{y_{-}}^{y_{+}} \mathrm{d} y \frac{y^{2}}{\left[y^{2} \mathcal{A}_{0}\left(\zeta^{\prime}, \gamma^{\prime}\right)+y\left(\mu^{2}+\gamma^{\prime}\right)+\mu^{2}\right]^{2}}\right] \\
\quad-\frac{\left(1+\zeta^{\prime}\right)}{(1+z)} \int_{0}^{\infty} \mathrm{d} y \frac{y^{2}}{\left[y^{2} \mathcal{A}_{0}\left(\zeta^{\prime}, \gamma^{\prime}\right)+y\left(\mu^{2}+\gamma^{\prime}\right)+\mu^{2}\right]^{2}},
\end{aligned}
$$

with [see also Eq. (D.37)]

$$
\begin{aligned}
& \mathcal{B}_{0}\left(z, \zeta^{\prime}, \gamma^{\prime}, \gamma^{\prime \prime}, \mu^{2}\right)=\mu^{2}+\gamma^{\prime}-\gamma^{\prime \prime} \frac{\left(1+\zeta^{\prime}\right)}{(1+z)} \leq 0, \\
& \Delta_{0}^{2}\left(z, \zeta^{\prime}, \gamma^{\prime}, \gamma^{\prime \prime}, \mu^{2}\right)= \\
& \quad=\mathcal{B}_{0}^{2}\left(z, \zeta^{\prime}, \gamma^{\prime}, \gamma^{\prime \prime}, \mu^{2}\right)-4 \mu^{2} \mathcal{A}_{0}\left(\zeta^{\prime}, \gamma^{\prime}\right) \geq 0, \\
& y_{ \pm}=\frac{1}{2 \mathcal{A}_{0}\left(\zeta^{\prime}, \gamma^{\prime}\right)} \\
& \quad \times\left[-\mathcal{B}_{0}\left(z, \zeta^{\prime}, \gamma^{\prime}, \gamma^{\prime \prime}, \mu^{2}\right) \pm \Delta_{0}\left(z, \zeta^{\prime}, \gamma^{\prime}, \gamma^{\prime \prime}, \mu^{2}\right)\right] .
\end{aligned}
$$

Notice that in the inhomogeneous term in Eq. (D.38) the factor $\theta\left(\mu^{2}-\gamma^{\prime \prime}\right)$ has been dropped, given the presence of the step functions $\theta\left[1 \pm z-\gamma^{\prime \prime} / \mu^{2}\right]$. In Eq. (103) of Ref. [9] the step function $\theta\left(\mu^{2}-\gamma^{\prime \prime}\right)$ has been accidentally overlooked.

In conclusion, by applying the Nakanishi theorem on the uniqueness of the weight function [12], one has the following integral equation:

$$
\begin{aligned}
& g_{0 L d}^{(+)}(\gamma, z)=\frac{g^{2}}{\mu^{2}} \theta(\gamma) \theta\left[\mu^{2}(1-|z|)-\gamma\right] \\
& \quad-\frac{g^{2}}{2(4 \pi)^{2}} \theta(\gamma) \int_{0}^{\infty} \mathrm{d} \gamma^{\prime} \int_{-1}^{1} \mathrm{~d} z^{\prime} g_{0 L d}^{(+)}\left(\gamma^{\prime}, z^{\prime}\right) \\
& \quad \times\left[\frac{(1+z)}{\left(1+z^{\prime}\right)} \theta\left(z^{\prime}-z\right) h_{0}^{\prime}\left(\gamma, z ; \gamma^{\prime}, z^{\prime}, \mu^{2}\right)\right. \\
& \left.\quad+\frac{(1-z)}{\left(1-z^{\prime}\right)} \theta\left(z-z^{\prime}\right) h_{0}^{\prime}\left(\gamma,-z ; \gamma^{\prime},-z^{\prime}, \mu^{2}\right)\right] .
\end{aligned}
$$

\section{Appendix E: An effective decomposition of $g_{0 L d}^{(+)}(\gamma, z)$}

In this appendix, the decomposition of $g_{0 L d}^{(+)}(\gamma, z)$ shown in Eq. (56) is applied to the simple case of Eq. (53), based on the Nakanishi uniqueness theorem [12], in order to give the explicit representation of the numerical system to be solved.

Inserting the decomposition in Eq. (56),

$g_{0 L d}^{(+)}(\gamma, z)=\beta \theta(-t)+\theta(t) \sum_{\ell=0}^{N_{z}} \sum_{j=0}^{N_{g}} A_{\ell j} G_{\ell}(z) \mathcal{L}_{j}(t)$,

with $t=\gamma-\mu^{2}(1-|z|)$, in Eq. (53), given by (notice that in the following expression, the symmetry properties of both the weight function and the kernel $h_{0}^{\prime}$ are exploited)

$$
\begin{gathered}
g_{0 L d}^{(+)}(\gamma, z)=\frac{g^{2}}{\mu^{2}} \theta(\gamma) \theta\left[\mu^{2}(1-|z|)-\gamma\right] \\
-\frac{g^{2}}{(4 \pi)^{2}} \theta(\gamma) \int_{0}^{\infty} \mathrm{d} \gamma^{\prime} \int_{-1}^{1} \mathrm{~d} z^{\prime} g_{0 L d}^{(+)}\left(\gamma^{\prime}, z^{\prime}\right) \\
\quad \times \frac{(1+z)}{\left(1+z^{\prime}\right)} \theta\left(z^{\prime}-z\right) h_{0}^{\prime}\left(\gamma, z ; \gamma^{\prime}, z^{\prime}, \mu^{2}\right),
\end{gathered}
$$

one can quickly obtain the following coupled system:

$$
\begin{aligned}
& \beta \theta(-t)=\frac{g^{2}}{\mu^{2}} \theta(\gamma) \theta(-t)-\frac{g^{2}}{(4 \pi)^{2}} \theta(\gamma) \theta(-t) \beta \\
& \times \int_{-1}^{1} \mathrm{~d} z^{\prime} \int_{0}^{\mu^{2}\left(1-\left|z^{\prime}\right|\right)} \mathrm{d} \gamma^{\prime} \\
& \times \frac{(1+z)}{\left(1+z^{\prime}\right)} \theta\left(z^{\prime}-z\right) h_{0}^{\prime}\left(\gamma, z ; \gamma^{\prime}, z^{\prime}, \mu^{2}\right) \\
& -\frac{g^{2}}{(4 \pi)^{2}} \theta(\gamma) \theta(-t) \sum_{\ell=0}^{N_{z}} \sum_{j=0}^{N_{g}} A_{\ell j} \int_{-1}^{1} \mathrm{~d} z^{\prime}
\end{aligned}
$$




$$
\begin{aligned}
\times & \int_{\mu^{2}\left(1-\left|z^{\prime}\right|\right)}^{\infty} \mathrm{d} \gamma^{\prime} G_{\ell}\left(z^{\prime}\right) \mathcal{L}_{j}\left[\gamma^{\prime}-\mu^{2}\left(1-\left|z^{\prime}\right|\right)\right] \\
\times & \frac{(1+z)}{\left(1+z^{\prime}\right)} \theta\left(z^{\prime}-z\right) h_{0}^{\prime}\left(\gamma, z ; \gamma^{\prime}, z^{\prime}, \mu^{2}\right) \\
\theta(t) & \sum_{\ell=0}^{N_{z}} \sum_{j=0}^{N_{g}} A_{\ell j} G_{\ell}(z) \mathcal{L}_{j}(t) \\
= & -\frac{g^{2}}{(4 \pi)^{2}} \theta(\gamma) \theta(t) \beta \int_{-1}^{1} \mathrm{~d} z^{\prime} \int_{0}^{\mu^{2}\left(1-\left|z^{\prime}\right|\right)} \mathrm{d} \gamma^{\prime} \\
& \times \frac{(1+z)}{\left(1+z^{\prime}\right)} \theta\left(z^{\prime}-z\right) h_{0}^{\prime}\left(\gamma, z ; \gamma^{\prime}, z^{\prime}, \mu^{2}\right)+ \\
& -\frac{g^{2}}{(4 \pi)^{2}} \theta(\gamma) \theta(t) \sum_{\ell=0}^{N_{z}} \sum_{j=0}^{N_{g}} A_{\ell j} \int_{-1}^{1} \mathrm{~d} z^{\prime} \\
& \int_{\mu^{2}\left(1-\left|z^{\prime}\right|\right)}^{\infty} \mathrm{d} \gamma^{\prime} G_{\ell}\left(z^{\prime}\right) \mathcal{L}_{j}\left[\gamma^{\prime}-\mu^{2}\left(1-\left|z^{\prime}\right|\right)\right] \\
& \times \frac{(1+z)}{\left(1+z^{\prime}\right)} \theta\left(z^{\prime}-z\right) h_{0}^{\prime}\left(\gamma, z ; \gamma^{\prime}, z^{\prime}, \mu^{2}\right) .
\end{aligned}
$$

$$
\begin{gathered}
I_{\ell^{\prime} j^{\prime}, \beta}=-\frac{g^{2}}{(4 \pi)^{2}} \int_{-1}^{1} \mathrm{~d} z \int_{0}^{\infty} d t G_{\ell^{\prime}}(z) \mathcal{L}_{j^{\prime}}(t) \int_{z}^{1} \mathrm{~d} z^{\prime} \\
\times \int_{0}^{\mu^{2}\left(1-\left|z^{\prime}\right|\right)} \mathrm{d} \gamma^{\prime} \frac{(1+z)}{\left(1+z^{\prime}\right)} h_{0}^{\prime}\left(\gamma, z ; \gamma^{\prime}, z^{\prime}, \mu^{2}\right)
\end{gathered}
$$$$
\text { where (cf. Eq. (51)) }
$$

and

$$
\begin{aligned}
& I_{\ell^{\prime} j^{\prime}, \ell j}=-\frac{g^{2}}{(4 \pi)^{2}} \int_{-1}^{1} \mathrm{~d} z \int_{0}^{\infty} d t G_{\ell^{\prime}}(z) \mathcal{L}_{j^{\prime}}(t) \\
& \int_{z}^{1} \mathrm{~d} z^{\prime} \int_{\mu^{2}\left(1-\left|z^{\prime}\right|\right)}^{\infty} \mathrm{d} \gamma^{\prime} G_{\ell}\left(z^{\prime}\right) \\
& \quad \times \mathcal{L}_{j}\left[\gamma^{\prime}-\mu^{2}\left(1-\left|z^{\prime}\right|\right)\right] \frac{(1+z)}{\left(1+z^{\prime}\right)} h_{0}^{\prime}\left(\gamma, z ; \gamma^{\prime}, z^{\prime}, \mu^{2}\right) .
\end{aligned}
$$

\section{References}

1. E.E. Salpeter, H.A. Bethe, A relativistic equation for bound-State problems. Phys. Rev. 84, 1232 (1951)

For $\gamma=0$ and $z=0$, Eq. (E.47) reduces to

$\beta=\frac{g^{2}}{\mu^{2}}+\beta I_{\beta, \beta}+\sum_{\ell=0}^{N_{z}} \sum_{j=0}^{N_{g}} I_{\beta, \ell j} A_{\ell j}$,

where

$$
\begin{gathered}
I_{\beta \beta}=-\frac{g^{2}}{(4 \pi)^{2}} \int_{0}^{1} \mathrm{~d} z^{\prime} \int_{0}^{\mu^{2}\left(1-z^{\prime}\right)} \mathrm{d} \gamma^{\prime} \\
\times \frac{1}{\left(1+z^{\prime}\right)} h_{0}^{\prime}\left(\gamma=0, z=0 ; \gamma^{\prime}, z^{\prime}, \mu^{2}\right)
\end{gathered}
$$

and

$$
\begin{aligned}
& I_{\beta, \ell j}=-\frac{g^{2}}{(4 \pi)^{2}} \\
& \quad \times \int_{0}^{1} \mathrm{~d} z^{\prime} \int_{\mu^{2}\left(1-z^{\prime}\right)}^{\infty} \mathrm{d} \gamma^{\prime} G_{\ell}\left(z^{\prime}\right) \mathcal{L}_{j}\left[\gamma^{\prime}-\mu^{2}\left(1-z^{\prime}\right)\right] \\
& \times \frac{1}{\left(1+z^{\prime}\right)} h_{0}^{\prime}\left(\gamma=0, z=0 ; \gamma^{\prime}, z^{\prime}, \mu^{2}\right)
\end{aligned}
$$

with (cf. Eq. (51))

$$
\begin{aligned}
& h_{0}^{\prime}\left(\gamma=0, z=0 ; \gamma^{\prime}, z^{\prime}, \mu^{2}\right)=-\left(1+z^{\prime}\right) \\
& \quad \times \int_{0}^{\infty} \mathrm{d} y \frac{y^{2}}{\left[y^{2}\left(\gamma^{\prime}+m^{2} z^{\prime 2}\right)+y\left(\gamma^{\prime}+\mu^{2}\right)+\mu^{2}\right]^{2}} .
\end{aligned}
$$

A matrix representation can be obtained for Eq. (E.48) by multiplying both sides by $G_{\ell^{\prime}}(z) \mathcal{L}_{j^{\prime}}(t)$ and integrating, viz.

$$
A_{\ell^{\prime} j^{\prime}}=\beta I_{\ell^{\prime} j^{\prime}, \beta}+\sum_{\ell=0}^{N_{z}} \sum_{j=0}^{N_{g}} I_{\ell^{\prime} j^{\prime}, \ell j} A_{\ell j}
$$

2. K. Kusaka, A.G. Williams, Solving the Bethe-Salpeter equation for scalar theories in Minkowski space. Phys. Rev. D 51, 7026 (1995)

3. K. Kusaka, K. Simpson, A.G. Williams, Solving the BetheSalpeter equation for bound states of scalar theories in Minkowski space. Phys. Rev. D 56, 5071 (1997) Minkowski space. Eur. Phys. J. A 27, 1 (2006)

5. J. Carbonell, V.A. Karmanov, Cross-ladder effects in BetheSalpeter and light-front equations. Eur. Phys. J. A 27, 11 (2006)

6. J. Carbonell, V.A. Karmanov, M. Mangin-Brinet, Electromagnetic form factors via Bethe-Salpeter amplitude in Minlowski space. Eur. Phys. J. A 39, 53 (2009)

7. J. Carbonell, V.A. Karmanov, Solutions of the Bethe-Salpeter equation in Minkowski space and applications to electromagnetic form factors. Few-body Syst. 49, 205 (2011)

8. J. Carbonell, V.A. Karmanov, Solving the Bethe-Salpeter equation for two fermions in Minkowski space. Eur. Phys. J. A 46, 387 (2010)

9. T. Frederico, G. Salmè, M. Viviani, Two-body scattering states in Minkowski space and the Nakanishi integral representation onto the null plane. Phys. Rev. D 85, 036009 (2012)

10. T. Frederico, G. Salmè, M. Viviani, Quantitative studies of the homogeneous Bethe-Salpeter equation in Minkowski space. Phys. Rev. D 89, 016010 (2014)

11. T. Frederico, G. Salmè, M. Viviani, Solutions of the Bethe-Salpeter equation in Minkowski space: a comparative study. Few-Body Sys. 55, 693 (2014)

12. N. Nakanishi, Graph Theory and Feynman Integrals (Gordon and Breach, New York, 1971)

13. J. Carbonell, V.A. Karmanov, Bethe-Salpeter scattering amplitude in Minkowski space. Phys. Lett. B 727, 319 (2013)

14. J. Carbonell, V.A. Karmanov, Bethe-Salpeter scattering state equation in Minkowski space. Phys. Rev. D 90, 056002 (2014)

15. P.C. Magalhães, M.R. Robilotta, K.S.F.F. Guimarães, T. Frederico, W. de Paula, I. Bediaga, A.C. dos Reis, C.M. Maekawa, G.R.S. Zarnauskas, Towards three-body unitarity in $D^{+} \rightarrow K^{-} \pi^{+} \pi^{-}$. Phys. Rev. D 84, 094001 (2011)

16. K.S.F.F. Guimarães, O. Lourenço, W. de Paula, T. Frederico, A.C. dos Reis, Final state interaction in $D^{+} \rightarrow K^{-} \pi^{+} \pi^{+}$with $K \pi I=$ $1 / 2$ and 3/2 channels. J. High Energy Phys. 1408, 135 (2014)
4. V.A. Karmanov, J. Carbonell, Solving Bethe-Salpeter equation in 
17. I. Bediaga, T. Frederico, O. Lourenço, $\mathrm{CP}$ violation and CPT invariance in $B^{ \pm}$decays with final state interactions. Phys. Rev. D 89, 094013 (2014)

18. T. Frederico, G. Salmè, M. Viviani (in preparation)

19. C. Itzykson, J.B. Zuber, Quantum Field Theory (Dover Publications, New York, 2006)

20. G.C. Wick, Properties of Bethe-Salpeter wave functions. Phys. Rev. 96, 1124 (1954)

21. R.E. Cutkosky, Solutions of a Bethe-Salpeter equation. Phys. Rev. 96, 1135 (1954)

22. S.J. Brodsky, H.C. Pauli, S.S. Pinsky, Quantum chromodynamics and other field theories on the light cone. Phys. Rep. 301, 299 (1998)
23. J. Carbonell, B. Desplanques, V.A. Karmanov, J.F. Mathiot, Explicitly covariant light-front dynamics and relativistic few-body systems. Phys. Rep. 300, 215 (1998)

24. S. Weinberg, Quasiparticles and the Born series. Phys. Rev. 131, $440(1963)$

25. H. Klar, H. Krüger, Approximate construction of the scattering amplitude from Mandelstam representation and elastic unitarity. Zeit. Phys. 194, 89 (1966)

26. V. Gigante, T. Frederico, C. Gutierrez, L. Tomio, Bound states in Minkowski space in $2+1$ dimensions. Few-Body Syst. doi:10. 1007/s00601-015-0986-8 